Copyright (C) 2005 IEEE. Reprinted from

IEEE Transactions on Information Theory, 2005; 51 (2):620-633

This material is posted here with permission of the IEEE. Such permission of the IEEE does not in any way imply IEEE endorsement of any of the University of Adelaide's products or services. Internal or personal use of this material is permitted. However, permission to reprint/republish this material for advertising or promotional purposes or for creating new collective works for resale or redistribution must be obtained from the IEEE by writing to pubs-permissions@ieee.org.

By choosing to view this document, you agree to all provisions of the copyright laws protecting it. 


\title{
Updating the Parameters of a Threshold Scheme by Minimal Broadcast
}

\author{
S. G. Barwick, Wen-Ai Jackson, and Keith M. Martin
}

\begin{abstract}
Threshold schemes allow secret data to be protected among a set of participants in such a way that only a prespecified threshold of participants can reconstruct the secret from private information (shares) distributed to them on a system setup using secure channels. We consider the general problem of designing unconditionally secure threshold schemes whose defining parameters (the threshold and the number of participants) can later be changed by using only public channel broadcast messages. In this paper, we are interested in the efficiency of such threshold schemes, and seek to minimize storage costs (size of shares) as well as optimize performance in low-bandwidth environments by minimizing the size of necessary broadcast messages. We prove a number of lower bounds on the smallest size of broadcast message necessary to make general changes to the parameters of a threshold scheme in which each participant already holds shares of minimal size. We establish the tightness of these bounds by demonstrating optimal schemes.
\end{abstract}

Index Terms-Cryptology, secret-sharing schemes, threshold schemes.

\section{INTRODUCTION}

$\mathbf{L}$ ET $k$ and $n$ be integers satisfying $1 \leq k \leq n$. A $(k, n)$ threshold scheme [3], [17] is a system for sharing a piece of secret information, known as the secret, among a set $\mathcal{P}$ of $n$ participants in such a way that the secret can be reconstructed from any $k$ shares, where a share is a private piece of information distributed securely by a trusted dealer to each participant on initial setup of the threshold scheme. The threshold structure $\Gamma$ is the collection of subsets of $\mathcal{P}$ whose shares can collectively be used to reconstruct the secret, in other words

$$
\Gamma=\{A \subseteq \mathcal{P}:|A| \geq k\} .
$$

All the threshold schemes discussed in this paper are perfect in the sense that knowledge of $k-1$ shares contributes no information to knowledge of the secret, and unconditionally secure in the sense that the security of the system does not depend on the difficulty of factorization, etc.

Threshold schemes are useful cryptographic primitives with many different applications. Examples include access control, protection of a cryptographic key, group signature protocols, and controlled key recovery. All these applications have in

Manuscript received July 3, 2003; revised November 4, 2004. This work was supported by the Australian Research Council.

S. G. Barwick and W.-A. Jackson are with the Department of Pure Mathematics, University of Adelaide, Adelaide 5005, Australia

K. M. Martin is with the Information Security Group, Royal Holloway, University of London, Egham, Surrey TW20 0EX, U.K.

Communicated by T. Johansson, Associate Editor for Complexity and Cryptography.

Digital Object Identifier 10.1109/TIT.2004.840857 common the need to distribute trust in a secret parameter among a number of different entities. For more details of some applications see, for example, [19].

There is a significant communication cost involved in setting up a $(k, n)$-threshold scheme since the dealer must use secure channels to distribute each participant's share to them. There are many applications where such a one-off cost can be tolerated, but where it is not practical to assume the existence of such secure channels after the setup process has completed. For example, root cryptographic keys are often protected by threshold schemes where shares of the key are distributed manually to participants. This manual distribution process represents a temporary secure channel between the dealer and each participant that may not be practical to reactivate at a later date (the participants might be based in different countries, for example).

This raises the interesting question as to whether it is possible to make changes to the basic parameters of a $(k, n)$-threshold scheme after the setup process has completed without having to use secure channels. Such a change may be required for a number of reasons: a set of participants might need to be removed from the scheme (disenrollment), involving a reduction in $n$; a set of participants might need to be added to the scheme (enrollment), involving an increase in $n$; the security policy relating to the threshold scheme might need to be strengthened (threshold increase), involving an increase in $k$; or slackened (threshold decrease), involving a decrease in $k$; or indeed any combination of the above.

An impractical solution to this problem would be for the dealer to distribute to each participant at setup not only a share in the original $(k, n)$-threshold scheme, but also one share in every possible $\left(k^{\prime}, n^{\prime}\right)$ threshold scheme that might be required in the future. To change parameters it would suffice that the dealer use a public channel to broadcast a message instructing participants to start using the appropriate new shares. However, this solution generally requires each participant to store an excessive number of unnecessary shares.

It is, therefore, desirable to investigate threshold schemes where participants do not hold excessively large shares (we will be interested in them holding shares of minimal size), but where the dealer can still use a public channel to broadcast some information that enables the threshold scheme parameters to change. Each participant in the "new" threshold scheme can determine their share exclusively from the information that they received on system setup and the broadcast message. We assume that the dealer anticipates that a future parameter change may be necessary before issuing the initial shares. This allows the dealer to build the capability for parameter change into the threshold scheme at setup. Without this assumption 
there are only a few types of parameter change that can be enabled using only broadcast channels (see Section VI).

The schemes that we look at will vary depending on the amount of knowledge that the dealer has about what future parameter changes will be needed. For reasons that we make clear in Section III, the following three cases are of particular interest.

- The dealer anticipates that the threshold may decrease (but not by how much) and that some participants may need to be disenrolled (but does not know how many).

- The dealer anticipates that the threshold may increase (but not by how much) and that some participants may need to be disenrolled (but does not know how many).

- The dealer anticipates that the threshold may change (but does not know whether it will increase or decrease) and that some participants may need to be disenrolled (but does not know how many).

Threshold schemes capable of changing their parameters within the same communications network context as this paper have been studied by a number of authors. A lower bound on the necessary share size to enable sequential disenrollment of participants in a threshold scheme was given in [4]. Both [4] and [15] demonstrated the optimality of this bound by providing different threshold schemes that met this lower share bound. In [5], a framework was provided for studying this problem for secret sharing schemes (a generalization of threshold schemes) and the lower bound on share size proved in [4] was generalized for this environment.

We are interested in not just minimizing the share size, but also the necessary broadcast information to enable a change in the parameters of a threshold scheme. In [2], a lower bound was shown for the amount of broadcast information necessary in the sequential disenrollment schemes of [4], [15]. In this paper, we significantly extend this work by looking at general parameter changes for threshold schemes. In particular, we will provide lower bounds on the size of broadcast message necessary to enable any type of meaningful change to the parameters of a threshold scheme that already has minimal share size. The following is a simplified version of our main theorem (Theorem 15).

Theorem: Consider a $(k, n)$-threshold scheme with $k<n$ on a participant set $\mathcal{P}$ with a secret $s$ to be updated via a broadcast to $\Gamma^{\prime}$, any $\left(k^{\prime}, n^{\prime}\right)$-threshold access structure with secret $s^{\prime}$ on a subset of $\mathcal{P}$. Let $b_{\Gamma^{\prime}}$ be the associated broadcast. Suppose that each participant $p$ holds a share of minimal size (that is, $\left.H(p)=2 H(s)=2 H\left(s^{\prime}\right)\right)$. Then, the size $H\left(b_{\Gamma^{\prime}}\right)$ of the broadcast $b_{\Gamma^{\prime}}$ satisfies

$$
\begin{aligned}
& H\left(b_{\Gamma^{\prime}}\right) \geq \begin{cases}\left(\min \left(n-1, n^{\prime}\right)-k^{\prime}+1\right) H(s) & \text { in case }(a) \\
\left(n^{\prime}-k^{\prime}+1\right) H(s) & \text { in case }(b) \\
\left(\min \left(k, n^{\prime}\right)-k^{\prime}+1\right) H(s) & \text { in case }(c)\end{cases} \\
& \text { case }(a) \text { is: } k^{\prime} \geq k \text { and } n^{\prime} \leq n
\end{aligned}
$$

We also show that these bounds are optimal by exhibiting schemes that meet these bounds.

Note that our communications environment differs from the one on which the redistribution techniques of [7], [9], [16] can be used to change the parameters of a threshold scheme. In redistribution environments there is no secure channel from the dealer to the participants to enable parameter change, but there do exist secure channels between the participants themselves. We make no such assumption here. The problem under discussion here is also related, but different, to the concept of proactive threshold schemes [10], where broadcast messages are used to refresh shares, but not to change the parameters of the threshold scheme.

The remainder of the paper is structured as follows. In Section II, we introduce the necessary preliminary concepts about threshold schemes. In Section III, we present the model we use and discuss the methodology of the paper. In Section IV, we present some preliminary results using the model for dynamic threshold schemes. In Sections V-VII, we consider three different types of parameter change and establish lower bounds on the broadcast size for share-minimal threshold schemes enabling such changes to be made. We establish the optimality of these bounds in Section VIII by demonstrating optimal constructions of dynamic threshold schemes and present a small example to illustrate the construction. In Section IX, we discuss two possible avenues for further work. Appendix I contains the proofs of Theorems 12 and 14.

\section{PRELIMINARIES}

Since the threshold schemes that we discuss here are unconditionally secure (their security is independent of cryptographic assumptions on the strength of an adversary), we follow the popular convention (first proposed by [14]) of modeling them in information-theoretic terms. We will formally define a threshold scheme within this context.

\section{A. Introduction to Information Theory}

We provide a short introduction to entropy here, but refer the reader to, for example, [8] for details.

For ease of translation from sets to random variables, throughout this paper we adopt the following conventions: if $A$ and $B$ are finite sets then we simplify $A \cup B$ to $A B$ and the singleton set $\{x\}$ to $x$ (hence, $s \mathcal{P}$ represents the set $\{s\} \cup \mathcal{P}$, etc.).

Let $X$ be a finite set. Let $\langle X\rangle$ be a finite collection of tuples, such that the entries of a tuple $\pi \in\langle X\rangle$ are indexed by the elements of $X$. Let $\rho$ be a probability distribution on $\langle X\rangle$. For $\pi=\left(\pi_{x}\right)_{x \in X} \in\langle X\rangle$ and $A \subseteq X$, let $\pi_{A}=\left(\pi_{x}\right)_{x \in A}$ and let $\langle A\rangle=\left\{\pi_{A} \mid \pi \in X\right\}$. Let $\rho_{A}$ be the marginal distribution on $A$, that is, $\rho_{A}$ is the probability distribution on $\langle A\rangle$ such that for $\alpha \in\langle A\rangle$ we have

$$
\rho_{A}(\alpha)=\sum_{\left\{\pi \in\langle X\rangle \mid \pi_{A}=\alpha\right\}} \rho(\pi) .
$$

Let $[A]_{\rho}=\left\{\alpha \in\langle A\rangle \mid \rho_{A}(\alpha)>0\right\}$. We use the notation $(X, \rho)$ to denote the set of tuples $[X]_{\rho}$ indexed by $X$ with the associated probability distribution $\rho$. 
The entropy $H_{\rho}(A)$ of $\rho_{A}$ is defined to be

$$
H_{\rho}(A)=-\sum_{\alpha \in[A]_{\rho}} \rho_{A}(\alpha) \log \rho_{A}(\alpha)
$$

We remark that the base of the logarithm is not specified here, but can be chosen to be any convenient value. Where there is no ambiguity, we will write $[A]$ for $[A]_{\rho}$ and $H$ for $H_{\rho}$. Let $B \subseteq X$ and let $\beta \in[B]$. For $\alpha \in[A]$, we have the conditional probability

$$
\rho_{A \mid B}(\alpha, \beta)=\frac{\sum_{\left\{\pi \in\langle X\rangle \mid \pi_{A}=\alpha, \pi_{B}=\beta\right\}} \rho(\pi)}{\rho_{B}(\beta)} .
$$

We may write $\rho_{A \mid B=\beta}$ for $\rho_{A \mid B}(\alpha, \beta)$, so we can regard $\rho_{A \mid B=\beta}$ as a probability distribution on $[A]_{\rho}$. The conditional entropy $H(A \mid B=\beta)$ of $\rho_{A \mid B=\beta}$ is defined to be

$$
H(A \mid B=\beta)=-\sum_{\alpha \in[A]} \rho_{A \mid B}(\alpha, \beta) \log \rho_{A \mid B}(\alpha, \beta) .
$$

The conditional entropy $H(A \mid B)$ of $\rho_{A}$ given $\rho_{B}$ is defined to be

$$
H(A \mid B)=\sum_{\beta \in[B]} H(A \mid B=\beta) \rho_{B}(\beta)
$$

and it can be shown that

$$
H(A \mid B)=H(A B)-H(B) .
$$

Note that if $H(A \mid B)=H(A)$ then $A$ and $B$ are independent variables and so $\rho_{A \mid B}(\alpha, \beta)=\rho_{A}(\alpha)$. Hence, $H(A \mid B)=$ $H(A)$ implies that $H(A \mid B=\beta)=H(A)$.

For $C \subseteq X$, the mutual information $I(A ; B \mid C)$ of $\rho_{A}$ and $\rho_{B}$ given $\rho_{C}$ is defined to be

$$
I(A ; B \mid C)=H(A \mid C)-H(A \mid B C)
$$

and so

$$
I(A ; B \mid C)=I(B ; A \mid C) .
$$

If $C=\emptyset$, we write $I(A ; B)$ for $I(A ; B \mid \emptyset)$. The following inequalities can be shown:

$$
\begin{aligned}
I(A ; B \mid C) & \geq 0 \\
H(A) \geq H(A \mid B) & \geq H(A \mid B C) \geq 0 \\
H(A) & \geq I(A ; B \mid C) .
\end{aligned}
$$

\section{B. Threshold Schemes}

Let $\mathcal{P}=\left\{p_{1}, \ldots, p_{n}\right\}$ be a set of participants, let $s$ be the secret, and let $k$ be an integer with $1 \leq k \leq n$.

Definition 1: A $(k, n)$-threshold scheme $\mathcal{M}=(s \mathcal{P}, \rho)$ is a probability distribution $\rho$ defined on a collection of tuples $\langle s \mathcal{P}\rangle$, each of which is indexed by the elements of $s \mathcal{P}$, such that for $A \subseteq \mathcal{P}$

$$
H(s \mid A)= \begin{cases}0, & \text { if }|A| \geq k \\ H(s), & \text { if }|A| \leq k-1 .\end{cases}
$$

We call the elements of $[s \mathcal{P}]$ distribution rules. Note that these are the tuples of $\langle s \mathcal{P}\rangle$ that occur with nonzero probability. In order to implement a threshold scheme, the collection $[s \mathcal{P}]$ of distribution rules is made public. A dealer privately selects a distribution rule $\pi=\left(x_{s}, x_{1}, \ldots, x_{n}\right)$ with probability $\rho(\pi)$, then securely distributes $x_{i}$ as a share to $p_{i}$, for $i=1, \ldots, n$. The element $x_{s}$ is the secret, and is kept private.

We call $H\left(p_{i}\right)$ the size of the share associated with participant $p_{i}$, and $H(s)$ the size of the secret. It can be seen (for example, [20]) that in any threshold scheme $H\left(p_{i}\right) \geq H(s)$. If $H\left(p_{i}\right)=H(s)$ for all such $p_{i}$ then we say that the threshold scheme is ideal. Ideal $(k, n)$-threshold schemes can be found for all integers $1 \leq k \leq n$. We describe two examples that are used in Section VIII.

Example 2 (Shamir [17]): Let $\mathcal{P}=\left\{p_{1}, \ldots, p_{n}\right\}$, let $p$ be a prime, and let $\mathcal{Z}_{p}$ be the field of integers modulo $p$. Suppose $k$ is an integer with $2 \leq k \leq n \leq p$. A dealer generates distinct, nonzero elements $x_{1}, \ldots, x_{n}$ of $\mathcal{Z}_{p}$ and publishes them. The dealer then secretly and randomly chooses elements $a_{0}, a_{1}, \ldots, a_{k-1} \in \mathcal{Z}_{p}$ and forms the polynomial

$$
a(x)=a_{0}+a_{1} x+a_{2} x^{2}+\cdots+a_{k-1} x^{k-1} .
$$

For $i=1, \ldots, n$, the share $a\left(x_{i}\right)$ is issued to participant $p_{i}$ and the value of the secret is $a_{0}$. It is straightforward to verify that any $k$ participants can determine $a_{0}$ by polynomial interpolation, but any $k-1$ participants can obtain no information about the value of $a_{0}$, additional to the fact that it is in $\mathcal{Z}_{p}$. In this case, there are $p^{k}$ distribution rules $\left(a(0), a\left(x_{1}\right), \ldots, a\left(x_{n}\right)\right)$ in $[s \mathcal{P}]$, corresponding to the $p^{k}$ values of the $k$-tuple $\left(a_{0}, a_{1}, \ldots, a_{k-1}\right)$. Since $\rho$ is uniform, we have that $H\left(p_{i}\right)=H(s)=\log p$ and thus that the scheme is ideal.

Example 3: An equivalent way to construct an ideal $(k, n)$-threshold scheme uses a geometric construction in $\Sigma=\mathrm{PG}(k-1, q)$ (for a background in projective geometry see [11]). Let $\sigma: s \mathcal{P} \rightarrow \Sigma$ be a mapping that assigns to each participant $p_{i}$ as share a point $p_{i}^{\sigma}$ on a normal rational curve in $\Sigma$ and assigns the secret $s$ to be a further point $s^{\sigma}$ on this curve. If $k$ participants pool their shares, these shares span $\Sigma$ and so they can obtain the secret. If $k-1$ participants pool their shares, these shares span a $(k-2)$-dimensional subspace which contains no further point of the normal rational curve, so in particular does not contain $s^{\sigma}$. They thus have no information about the secret $s=s^{\sigma}$. To see how to extract the distribution rules of an ideal $(k, n)$-threshold scheme from this configuration of points see, for example, [13] or [20].

\section{Restrictions and Contractions}

In order to define our model rigorously, we will make use of two types of threshold schemes that can be derived from an existing threshold scheme. The restriction of a $(k, n)$-threshold scheme to a subset of $n^{\prime}$ participants is the $\left(k, n^{\prime}\right)$-threshold scheme that results from effectively discarding the shares held by the other $n-n^{\prime}$ participants. The contraction of a $(k, n)$-threshold scheme at a set of $r$ shares is the $(k-r, n-r)$-threshold scheme that results from effectively broadcasting this set of $r$ shares. 
More generally, let $\rho$ be a probability distribution on a finite collection $\langle X\rangle$ of tuples indexed by the finite set $X$. For $A \subseteq X$, the restriction to $A$ of the pair $(X, \rho)$ is the pair $\left(A, \rho_{A}\right)$. For $B \subseteq X$ and $A=X \backslash B$, the contraction at $B=\beta \in[B]$ of the pair $(X, \rho)$ is the pair $\left(A, \rho_{A \mid B=\beta}\right)$. Restrictions and contractions of threshold schemes are formalised by the following two results from [12].

Theorem 4 (Restriction): [12] Let $\mathcal{M}=(s \mathcal{P}, \rho)$ be a $(k, n)$-threshold scheme and let $\mathcal{P}^{\prime} \subseteq \mathcal{P}$. Then

$$
\mathcal{M}^{\prime}=\left(s \mathcal{P}^{\prime}, \rho_{s \mathcal{P}^{\prime}}\right)
$$

is a $\left(k,\left|\mathcal{P}^{\prime}\right|\right)$-threshold scheme, known as the restriction of $\mathcal{M}$ to $s \mathcal{P}^{\prime}$.

Theorem 5 (Contraction): [12] Let $\mathcal{M}=(s \mathcal{P}, \rho)$ be a $(k, n)$-threshold scheme. Let $B \subseteq \mathcal{P}$, let $\mathcal{P}^{\prime}=\mathcal{P} \backslash B$ and let $\beta \in[B]$. Then

$$
\mathcal{M}^{\prime}=\left(s \mathcal{P}^{\prime}, \rho_{s} \mathcal{P}^{\prime} \mid B=\beta\right)
$$

is a $\left(k-|B|,\left|\mathcal{P}^{\prime}\right|\right)$-threshold scheme, known as the contraction of $\mathcal{M}$ at $B=\beta$.

\section{DYNAMIC THRESHOLD SCHEMES}

In this paper, we are interested in threshold schemes where the parameters can later be changed by means of a public channel broadcast. In this section, we first comment on the special case of enrollment. We then propose a simple extension of the model of a threshold scheme within which to analyze dynamic threshold schemes.

\section{A. Enrollment}

There is one type of parameter change that cannot be easily accommodated in the communication network environment that we propose. Enrollment of new participants who were not issued with any private information at system setup is impossible in a broadcast-only network. The reason for this is that each new participant needs to acquire some private information from the dealer, which clearly needs the involvement at some stage of a secure distribution channel. The only ways in which enrollment could be performed are as follows.

- A secure channel is set up between the dealer and any new enrolling participants to issue them with shares.

- All possible future participants are issued with a cryptographic key at system setup. These participants are then effectively "sleeping participants" until the time of enrollment, when the dealer broadcasts their share to them, encrypted under the key that they were issued at setup. This is essentially the same technique used in [18] to remotely activate threshold schemes.

- Existing participants transfer necessary information using secure channels to new enrolling participants (this is outside our communications model, but is appropriate in the redistribution environments mentioned in Section I).
For this reason, we do not consider enrollment in the rest of this paper, and acknowledge that if enrollment is required then secure channels must be established using one of the above techniques.

\section{B. A Model for Dynamic Threshold Schemes}

Let $\mathcal{P}$ be a set of participants and $s$ be a secret. Let $\mathcal{U}$ be a collection of threshold structures defined on subsets of $\mathcal{P}$ (in other words, for each $\Gamma^{\prime} \in \mathcal{U}$ there exist $k^{\prime}, n^{\prime}$, and $\mathcal{P}^{\prime} \subseteq \mathcal{P}$ such that $\Gamma^{\prime}$ is a $\left(k^{\prime}, n^{\prime}\right)$-threshold structure defined on $\left.\mathcal{P}^{\prime}\right)$.

We wish to establish a $(k, n)$-threshold scheme defined on $\mathcal{P}$ that has the capability of being changed by means of a broadcast message into a scheme with threshold structure $\Gamma^{\prime}$, where $\Gamma^{\prime}$ can be any of the threshold structures in $\mathcal{U}$. We denote the secret after this change by $s^{\prime}$. We will also make the reasonable assumption throughout that

$$
H(s)=H\left(s^{\prime}\right) .
$$

Each $\Gamma^{\prime} \in \mathcal{U}$ is associated with a broadcast variable $b_{\Gamma^{\prime}}$, which represents the broadcast message that the dealer will send if he wishes to change to the threshold structure $\Gamma^{\prime}$. We let

$$
\mathcal{B}=\left\{b_{\Gamma^{\prime}} \mid \Gamma^{\prime} \in \mathcal{U}\right\}
$$

Definition 6: A $(k, n)$-threshold scheme $\mathcal{M}=\left(s s^{\prime} \mathcal{P B}, \rho\right)$ that can be updated to $\mathcal{U}$, is a probability distribution $\rho$ defined on a collection of tuples $\left\langle s s^{\prime} \mathcal{P B}\right\rangle$ (each of which is indexed by the elements of $\left.s s^{\prime} \mathcal{P B}\right)$ such that

(A) $\mathcal{M}$ restricted to $s \mathcal{P}$ is a $(k, n)$-threshold scheme on $\mathcal{P}$ with secret $s$, that is,

$$
H(s \mid A)= \begin{cases}0, & \text { if }|A| \geq k \\ H(s), & \text { if }|A| \leq k-1 .\end{cases}
$$

(B) For each threshold structure $\Gamma^{\prime} \in \mathcal{U}$, if $\Gamma^{\prime}$ is a $\left(k^{\prime}, n^{\prime}\right)$-threshold structure on $\mathcal{P}^{\prime}$ then $\mathcal{M}$ contracted at $b_{\Gamma^{\prime}}=\beta \in\left[b_{\Gamma^{\prime}}\right]$ is a $\left(k^{\prime}, n^{\prime}\right)$-threshold scheme on $\mathcal{P}^{\prime}$ with secret $s^{\prime}$, that is,

$$
H\left(s^{\prime} \mid A b_{\Gamma^{\prime}}\right)= \begin{cases}0, & \text { if }\left|A \cap \mathcal{P}^{\prime}\right| \geq k^{\prime} \\ H\left(s^{\prime}\right), & \text { if }\left|A \cap \mathcal{P}^{\prime}\right| \leq k^{\prime}-1 .\end{cases}
$$

In other words, $\mathcal{M}$ is initially a $(k, n)$-threshold scheme. If the broadcast message $b_{\Gamma^{\prime}}$ is sent on a public channel then knowledge of the original shares and this broadcast result in $\mathcal{M}$ also becoming a $\left(k^{\prime}, n^{\prime}\right)$-threshold scheme defined on $\mathcal{P}^{\prime}$.

Note that in Definition 6 we do not care whether a set of participants belonging to $\Gamma^{\prime}$ can obtain $s^{\prime}$ prior to knowledge of the broadcast $b_{\Gamma^{\prime}}$. It is foreseen that in most anticipated applications that $s^{\prime}$ will not have any relevant meaning until the point at which the dealer initiates a parameter change (for example, $s^{\prime}$ may be the backup master key that the dealer will only activate in the event that a parameter change is necessary).

We are interested first in minimizing the size of shares held by each participant, which is measured by $H(p)$. We are then interested in minimizing the size of the broadcast $b_{\Gamma^{\prime}}$, which is measured by $H\left(b_{\Gamma^{\prime}}\right)$. In this paper, for a number of different 


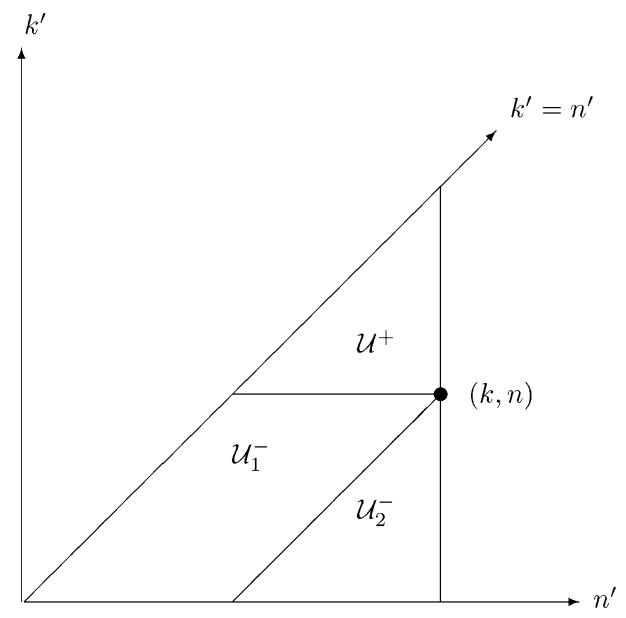

Fig. 1. Selected regions to which threshold schemes can be updated.

sets $\mathcal{U}$, we will determine the minimal size of broadcast necessary in a share minimal threshold scheme that can be updated to $\mathcal{U}$. Whereas lower bounds on share size are easily extracted from existing work and tend to be "expected," lower bounds on broadcast size are neither established nor particularly intuitive. For example, it would seem intuitive that broadcast size should depend on the set $\mathcal{U}$, and that larger sets $\mathcal{U}$ will require larger broadcasts, but we prove the slightly surprising result that the minimal broadcast size is "fairly independent" of $\mathcal{U}$.

We proceed by identifying some "sensible" sets $\mathcal{U}$ to study. First, observe that if a lower bound holds for updating to $\mathcal{U}$ then it also holds for updating to any region $\mathcal{U}^{\prime} \supseteq \mathcal{U}$. We thus identify some meaningful "large" regions $\mathcal{U}$ to investigate, and in Section VII-B will show that updating to "smaller" regions generally does not result in smaller broadcast sizes.

The three main regions $\mathcal{U}$ that we study are as follows.

1) Threshold increases. The dealer wants it to be possible to change to any threshold structure with a greater threshold parameter. In other words, all $\left(k^{\prime}, n^{\prime}\right)$-threshold structures with $k^{\prime} \geq k$ and $n^{\prime} \leq n$. We denote this set by $\mathcal{U}^{+}(k, n)$, or $\mathcal{U}^{+}$when no ambiguity arises.

2) Threshold decreases. The dealer wants it to be possible to change to any threshold structure with a smaller threshold parameter. In other words, all $\left(k^{\prime}, n^{\prime}\right)$-threshold structures with $k^{\prime} \leq k$ and $n^{\prime} \leq n$. We denote this set by $\mathcal{U}^{-}(k, n)$, or simply $\mathcal{U}^{-}$. For reasons that will later be explained, we partition $\mathcal{U}^{-}$into $\mathcal{U}_{1}^{-}$(corresponding to $k-k^{\prime}<n-n^{\prime}$ ) and $\mathcal{U}_{2}^{-}$(corresponding to $k-k^{\prime} \geq n-n^{\prime}$ ).

3) The general case. The dealer wants it to be possible to change to any $\left(k^{\prime}, n^{\prime}\right)$-threshold structure with $n^{\prime} \leq n$. We denote this set by $\mathcal{U}^{T}(k, n)$, or simply $\mathcal{U}^{T}$. Clearly, $\mathcal{U}^{T}=\mathcal{U}^{+} \cup \mathcal{U}^{-}=\mathcal{U}^{+} \cup \mathcal{U}_{1}^{-} \cup \mathcal{U}_{2}^{-}$.

An example of a smaller region of interest is where $k^{\prime}=k$ and $n^{\prime} \leq n$ (this corresponds to disenrollments). We return to this is Section VII-B. The listed regions are illustrated in Fig. 1.

A natural question to ask is: What is the relation between $s$ and $s^{\prime}$ ? We show in the next section that if $k^{\prime}>k$ (region $\left.\mathcal{U}^{+}\right)$ or if $\left(k^{\prime}<k\right.$ and) $k-k^{\prime} \geq n-n^{\prime}$ (region $\mathcal{U}_{1}^{-}$) then $s$ and $s^{\prime}$ are necessarily independent (Lemma 8 ). In the remaining case $k-k^{\prime} \geq n-n^{\prime}$ (region $\mathcal{U}_{2}^{-}$), we can have $s=s^{\prime}$. This is discussed in Section VI-A.

In the following sections we look at these different regions in turn and determine lower bounds on the broadcast size for updating to them. In Section V, we look at $\mathcal{U}^{+}$, in Section VI, we look at $\mathcal{U}^{-}$, and in Section VII, we look at $\mathcal{U}^{T}$ and discuss the possibility of improving these results for smaller update regions. We begin with some preliminary results.

\section{PReliminary Results For Dynamic THRESHOLD SCHEMES}

Let $\mathcal{M}=\left(s s^{\prime} \mathcal{P B}, \rho\right)$ be a $(k, n)$-threshold scheme with $k<$ $n$ that can be updated to $\mathcal{U}$, some collection of $\left(k^{\prime}, n^{\prime}\right)$-threshold structures with $n^{\prime} \leq n$. (The case $k=n$ is considered in Section VI-A and will not be considered further in this paper.) Let $\Gamma$ be the $(k, n)$ threshold structure on $\mathcal{P}$.

We first prove three lemmas that we will need later. The first lemma notes that for a large class of regions $\mathcal{U}$ the secrets $s$ and $s^{\prime}$ must be independent.

Lemma 7: If there exists $\Gamma^{\prime} \in \mathcal{U}$ with $\Gamma \not \subset \Gamma^{\prime}$ (that is, there exists a set $A \subseteq \mathcal{P}$ with $A \in \Gamma$ but $\left.A \notin \Gamma^{\prime}\right)$, then $H\left(s^{\prime} \mid s\right)=$ $H\left(s^{\prime}\right)$.

Proof: Suppose $\Gamma \nsubseteq \subset \Gamma^{\prime}$, so there exists a set $A \subseteq \mathcal{P}$ with $A \in \Gamma$ but $A \notin \Gamma^{\prime}$. Suppose $H\left(s^{\prime} \mid s\right)<H\left(s^{\prime}\right)$. As $H(s \mid A)=0$, we have

$$
H\left(s^{\prime} \mid A b_{\Gamma^{\prime}}\right)=H\left(s^{\prime} \mid A b_{\Gamma^{\prime}} s\right) \leq H\left(s^{\prime} \mid s\right)<H\left(s^{\prime}\right)
$$

contradicting (B) in Definition 6. Hence $H\left(s^{\prime} \mid s\right)=H\left(s^{\prime}\right)$.

Lemma 8: For $\Gamma^{\prime} \in \mathcal{U}^{+}$or $\mathcal{U}_{1}^{-}, s$ and $s^{\prime}$ are independent.

Proof: If $\Gamma^{\prime} \in \mathcal{U}^{+}$then $k^{\prime} \geq k$ so $\Gamma^{\prime} \nsubseteq \Gamma$ and Lemma 7 gives the result. Now consider $k^{\prime} \leq k$. Let $X=\mathcal{P} \backslash \mathcal{P}^{\prime}$. If $|X|>k$ then $|X| \geq k^{\prime}$ and so $X \in \Gamma^{\prime}$, contradicting the definition of $\Gamma^{\prime}$. Thus, $|X| \leq k$ and so there exists $A \subseteq \mathcal{P}^{\prime}$ with $|X A|=k$ so $|A|=k-\left(n-n^{\prime}\right)$. Now $X A \in \Gamma^{\prime}$ if and only if $\left|X A \cap \mathcal{P}^{\prime}\right| \geq k^{\prime}$, if and only if $|A| \geq k^{\prime}$, if and only if $k-\left(n-n^{\prime}\right) \geq k^{\prime}$, if and only if $k-k^{\prime} \geq n-n^{\prime}\left(\right.$ region $\left.\mathcal{U}_{2}^{-}\right)$. Thus, if $k-k^{\prime}<n-n^{\prime}$ (region $\mathcal{U}_{1}^{-}$) then $X A \notin \Gamma^{\prime}$ and so, by Lemma $7, s$ and $s^{\prime}$ are independent.

Note that in the region $\mathcal{U}_{2}^{-}$, it is possible to have $s=s^{\prime}$ and $H(p)=H(s)$. This is discussed in Section VI-A.

The next observation is an adaptation of a result in [6].

Lemma 9: If there exists $\Gamma^{\prime} \in \mathcal{U}, p \in \mathcal{P}$, and sets $X, X^{\prime} \subseteq \mathcal{P}$ with $X \notin \Gamma, p X \in \Gamma, X X^{\prime} \in \Gamma, X X^{\prime} \notin \Gamma^{\prime}, p X X^{\prime} \in \Gamma^{\prime}$, then $H(p) \geq H(s)+H\left(s^{\prime}\right)$.

Proof: We have

$$
\begin{aligned}
H(p) \geq & H(p \mid X) \text { by }(4) \\
= & H(s \mid X)-H(s \mid p X)+H(p \mid s X) \text { by (1) } \\
= & H(s)+H(p \mid s X) \text { as } X \notin \Gamma \text { and } p X \in \Gamma \\
\geq & H(s)+H\left(p \mid s X X^{\prime} b_{\Gamma^{\prime}}\right) \text { by (4) } \\
= & H(s)+H\left(s^{\prime} \mid s X X^{\prime} b_{\Gamma^{\prime}}\right)-H\left(s^{\prime} \mid p s X X^{\prime} b_{\Gamma^{\prime}}\right) \\
& +H\left(p \mid s s^{\prime} X X^{\prime} b_{\Gamma^{\prime}}\right) \text { by }(1) \\
= & H(s)+H\left(s^{\prime} \mid s X X^{\prime} b_{\Gamma^{\prime}}\right)+H\left(p \mid s s^{\prime} X X^{\prime} b_{\Gamma^{\prime}}\right) \\
& \text { as } p X X^{\prime} \in \Gamma^{\prime}
\end{aligned}
$$




$$
\begin{array}{ll}
=H(s)+H\left(s^{\prime}\right)+H\left(p \mid s s^{\prime} X X^{\prime} b_{\Gamma^{\prime}}\right) & \\
& \text { as } X X^{\prime} \in \Gamma \text { and } X X^{\prime} \notin \Gamma^{\prime} \\
\geq H(s)+H\left(s^{\prime}\right) \quad \text { by (4). }
\end{array}
$$

Lemma 10: Consider any probability distribution on a set $\langle Z\rangle$ with $s^{\prime}, p \in Z, X \subseteq Z$. Suppose $s^{\prime}, X$, and $p$ satisfy

$$
H\left(s^{\prime} \mid X\right)=H\left(s^{\prime}\right) \text { and } H\left(s^{\prime} \mid X p\right)=0 .
$$

Then $H(p \mid X) \geq H\left(s^{\prime}\right)$.

Proof:

$$
\begin{aligned}
H(p \mid X) & =H(p X)-H(X)=H\left(s^{\prime} p X\right)-H\left(s^{\prime} X\right)+H\left(s^{\prime}\right) \\
& =H\left(p \mid s^{\prime} X\right)+H\left(s^{\prime}\right) \geq H\left(s^{\prime}\right) .
\end{aligned}
$$

\section{INCREASING THE THRESHOLD}

In this section, we consider the case of increasing the threshold, that is, we look at updating to threshold structures in $\mathcal{U}^{+}$. We first need to establish exactly what the minimal share size is for this case.

Theorem 11: Let $\mathcal{M}=\left(s s^{\prime} \mathcal{P B}, \rho\right)$ be a $(k, n)$-threshold scheme with $k<n$ that can be updated to $\mathcal{U}^{+}$. Then

a) $s$ and $s^{\prime}$ are independent;

b) for each participant $p \in \mathcal{P}, H(p) \geq 2 H(s)$.

Proof: Let $\Gamma$ be a $(k, n)$-threshold structure on $\mathcal{P}$ with $k<$ $n$. Let $\Gamma^{\prime}$ be the $(n, n)$-threshold structure on $\mathcal{P}$, so $\Gamma^{\prime} \in \mathcal{U}^{+}$. Part a) follows from Lemma 8. For part b), for $p \in \mathcal{P}$, let $X \subseteq$ $\mathcal{P} \backslash p$ be a set of size $k-1$ and $X^{\prime}=\mathcal{P} \backslash(p X)$. Applying Lemma 9 and (6) we get $H(p) \geq H(s)+H\left(s^{\prime}\right)=2 H(s)$.

We thus refer to a $(k, n)$-threshold scheme that can be updated to $\mathcal{U}^{+}$and has $H(p)=2 H(s)$ for all $p \in \mathcal{P}$ as share minimal.

Theorem 12: Let $\mathcal{M}=\left(s s^{\prime} \mathcal{P} \mathcal{B}, \rho\right)$ be a share minimal $(k, n)$ threshold scheme with $k<n$ that can be updated to $\mathcal{U}^{+}(k, n)$. Then for any $\Gamma^{\prime} \in \mathcal{U}^{+}(k, n)$, where $\Gamma^{\prime}$ is a $\left(k^{\prime}, n^{\prime}\right)$-threshold structure

$$
H\left(b_{\Gamma^{\prime}}\right) \geq \begin{cases}\left(n^{\prime}-k^{\prime}+1\right) H(s), & \text { if } n^{\prime}<n \\ \left(n^{\prime}-k^{\prime}\right) H(s), & \text { if } n^{\prime}=n .\end{cases}
$$

The complete proof of this theorem is complex, see Appendix I-A for full details. We sketch the proof here to provide an idea of how it works. Theorem 12 is proved by induction on $k$. We first prove the result for $k=1$, that is, $\Gamma$ is an $(1, n)$ threshold scheme and $H(p)=2 H(s)=2 H\left(s^{\prime}\right)$. We derive two parts from $\mathcal{M}$, one part relating to $\Gamma$ and the other relating to $\Gamma^{\prime} \in \mathcal{U}^{+}(1, n)$. We then find entropy results concerning the participants, the broadcasts, and $s$ (see Lemmas 19 and 20 in Appendix I-A). Using these results, we can prove the size of the broadcast for $k=1$ (see Lemma 21 in Appendix I-A). To prove the result for general $k$, we assume that $k>1$, we contract on a $k-1$ subset of $\mathcal{P}$ to obtain a $(1, n-(k-1))$ threshold scheme that can be updated to $\mathcal{U}^{+}(1, n-(k-1))$ for an appropriate choice of broadcast. Now we apply the result for the case $k=1$ and Theorem 12 follows.

We will see in Section VIII that this bound is tight when we demonstrate a share minimal scheme that also has minimal broadcast size.
Recall that, as discussed immediately after Definition 6, our model is not concerned with whether participants belonging to $\Gamma^{\prime}$ can obtain $s^{\prime}$ prior to knowledge of the broadcast $b_{\Gamma^{\prime}}$. It is worth observing that if the model is restricted to make the extra requirement that they cannot obtain $s^{\prime}$, we get the simplified bound $H\left(b_{\Gamma^{\prime}}\right) \geq\left(n^{\prime}-k^{\prime}+1\right) H(s)$.

\section{DeCREASING THE Threshold}

In this section, we consider decreasing the threshold. We wish to establish a lower bound on the broadcast size necessary for a share minimal $(k, n)$-threshold scheme that can be updated to $\mathcal{U}^{-}$.

This case is interesting because there are a number of parameter sets within this region that any standard threshold scheme can be updated to, without the need for extra share information on scheme initialization. This partitions $\mathcal{U}^{-}$into two separate regions $\mathcal{U}_{1}^{-}$and $\mathcal{U}_{2}^{-}$, with $\mathcal{U}_{1}^{-}$corresponding to all $\left(k^{\prime}, n^{\prime}\right)$ pairs where $k-k^{\prime}<n-n^{\prime}$, and $\mathcal{U}_{2}^{-}$corresponds to all $\left(k^{\prime}, n^{\prime}\right)$ pairs where $k-k^{\prime} \geq n-n^{\prime}$.

We will first discuss a special case arising as a result of this issue and then deal with the two separate regions.

\section{A. A Special Case: Region $\mathcal{U}_{2}^{-}$}

The region $\mathcal{U}_{2}^{-}$is interesting because here $s$ and $s^{\prime}$ can be equal. It is possible to update any standard $(k, n)$-threshold scheme to a $\left(k^{\prime}, n^{\prime}\right)$-threshold scheme in $\mathcal{U}_{2}^{-}$by broadcasting some selected share information that allows remaining participants to continue using their original shares within a new scheme. A simple example would be the fact that broadcasting the share held by any participant effectively converts the original scheme into a $\left(k^{\prime}, n^{\prime}\right)$-threshold scheme, with that participant "removed." In such schemes there is no need to distribute extra share information and so $H(p) \geq H(s)$.

However, the region $\mathcal{U}_{2}^{-}$is otherwise artificial and providing the general capability of decreasing the threshold normally involves the inclusion of $\left(k^{\prime}, n^{\prime}\right)$ pairs outside this region, except in one significant case. When $k=n$, we have $\mathcal{U}_{1}^{-}=\emptyset$ and $\mathcal{U}_{2}^{-}=\mathcal{U}^{-}$. This special case was studied in [1], where it was shown that for schemes with the minimal share size of $H(p)=H(s)$, the minimal broadcast size is

$$
H\left(b_{\Gamma^{\prime}}\right)=\min \left(k-k^{\prime}, n^{\prime}-k^{\prime}+1\right) H(s) .
$$

We refer the reader to [1] for details, and for examples of schemes with this minimal broadcast size.

\section{B. Decreasing the Threshold When $k<n$}

For the rest of this section we thus only consider the case $k<n$. We first establish that in contrast to the special case $k=n$, when $k<n$ the size of shares in a $(k, n)$-threshold scheme that can be updated to $\mathcal{U}^{-}$is at least $2 H(s)$.

Theorem 13: Let $\mathcal{M}=\left(s s^{\prime} \mathcal{P B}, \rho\right)$ be a $(k, n)$-threshold scheme with $k<n$ that can be updated to $\mathcal{U}^{-}$. Then $H(p) \geq 2 H(s)$.

Proof: Let $\Gamma^{\prime} \in \mathcal{U}_{1}^{-}$be a $(1, n-k)$-threshold structure on $\mathcal{P}^{\prime}\left(\Gamma^{\prime}\right.$ exists as $k<n$ ). Noting that $\left|\mathcal{P} \backslash \mathcal{P}^{\prime}\right|=k$, choose $X \subseteq \mathcal{P} \backslash \mathcal{P}^{\prime}$ to be a set of size $k-1$ and $X^{\prime} \subseteq \mathcal{P} \backslash \mathcal{P}^{\prime}$ to be a set of size 1 disjoint from $X$. Now let $p \in \mathcal{P}^{\prime}$. The 
TABLE I

SHARE AND BROADCAST BOUNDS FOR UPDATING TO SOME SMALLER REGIONS $\mathcal{U}$

\begin{tabular}{|c|c|c|c|c|c|}
\hline \multirow[b]{2}{*}{ Case } & \multicolumn{3}{|c|}{$\mathcal{U}$ defined by } & \multirow{2}{*}{$\begin{array}{l}\text { Minimum } \\
\text { share size }\end{array}$} & \multirow{2}{*}{$\begin{array}{l}\text { Broadcast lower bound: } \\
H\left(b_{\Gamma^{\prime}}\right) \geq\end{array}$} \\
\hline & $k^{\prime}$ & $n^{\prime}$ & & & \\
\hline 1 & $k^{\prime}=k$ & $n^{\prime}=n-1$ & & $2 H(s)$ & $H(s)$ \\
\hline 2 & $k^{\prime}=k$ & $n^{\prime}<n$ & & $2 H(s)$ & $\left(n^{\prime}-k^{\prime}+1\right) H(s)$ \\
\hline 3 & $k^{\prime} \geq k$ & $n^{\prime}<n$ & & $2 H(s)$ & $\left(n^{\prime}-k^{\prime}+1\right) H(s)$ \\
\hline 4 & $k^{\prime} \leq k$ & $n^{\prime} \leq n$ & $k-k^{\prime}<n-n^{\prime}$ & $2 H(s)$ & $\left(n^{\prime}-k^{\prime}+1\right) H(s)$ \\
\hline 5 & $k^{\prime} \leq k$ & $n^{\prime} \leq n$ & $k-k^{\prime} \geq n-n^{\prime}$ & $H(s)$ & $\min \left(k-k^{\prime}, n^{\prime}-k^{\prime}+1\right) H(s)$ \\
\hline 6 & $k^{\prime}<k$ & $n^{\prime}=n$ & & $H(s)$ & $\min \left(k-k^{\prime}, n^{\prime}-k^{\prime}+1\right) H(s)$ \\
\hline
\end{tabular}

sets $p, X, X^{\prime}$ satisfy the conditions of Lemma 9 , so we have $H(p) \geq H(s)+H\left(s^{\prime}\right)=2 H(s)$ by $(6)$.

As in Section V, we refer to a scheme with the minimal share size of $H(p)=2 H(s)$ for all $p \in \mathcal{P}$ as share minimal. The main result of this section is the following.

Theorem 14: Let $\mathcal{M}=\left(s s^{\prime} \mathcal{P B}, \rho\right)$ be a share minimal $(k, n)$-threshold scheme with $k<n$ that can be updated to $\mathcal{U}^{-}$. Then for any $\Gamma^{\prime} \in \mathcal{U}^{-}$, where $\Gamma^{\prime}$ is a $\left(k^{\prime}, n^{\prime}\right)$-threshold structure

$$
H\left(b_{\Gamma^{\prime}}\right) \geq \begin{cases}\left(n^{\prime}-k^{\prime}+1\right) H(s), & \text { if } \Gamma^{\prime} \in \mathcal{U}_{1}^{-} \\ \left(\min \left(k, n^{\prime}\right)-k^{\prime}+1\right) H(s), & \text { if } \Gamma^{\prime} \in \mathcal{U}_{2}^{-}\end{cases}
$$

The complete proofs are in Appendix I-B, but we comment on them here. We have already seen that the two regions $\mathcal{U}_{1}^{-}$ and $\mathcal{U}_{2}^{-}$are in some respects fundamentally different. In fact, to prove a lower bound on the broadcast size for updating to $\mathcal{U}^{-}$we need two different approaches for each of these two regions. The method of proof for the region $\mathcal{U}_{1}^{-}$is similar to that of Theorem 12. The proof for the region $\mathcal{U}_{2}^{-}$involves the following series of steps. The first step is to show that, for $A \subseteq \mathcal{P}$ with $|A| \leq k, H(A)=2|A| H(s)$ and that for any $A \subseteq P$, $H(A)=(\min (k,|A|)+|A|) H(s)$. This is proved by induction on $k$. The next step is to prove that $H\left(b_{\Gamma^{\prime}}\right) \geq \min \left(k, n^{\prime}\right) H(s)$ if $\Gamma^{\prime}$ is a $\left(1, n^{\prime}\right)$ threshold structure in $\mathcal{U}_{2}^{-}(k, n)$. The final step is to prove the result for $\Gamma^{\prime} \in \mathcal{U}_{2}^{-}(k, n)$ by using induction on $k$.

\section{UPDATING TO OTHER REGIONS}

We have demonstrated lower bounds on the broadcast size of share minimal threshold schemes that can be updated to have lower, or higher threshold parameters. In this section, we briefly consider the general case, where the threshold can be either increased or decreased, and then discuss updating to smaller regions.

\section{A. Updating the Threshold to $\mathcal{U}^{T}$}

By Theorems 11 and 13, it follows that any $(k, n)$-threshold scheme with $k<n$ that can be updated to $\mathcal{U}^{T}$ has $H(p) \geq$ $2 H(s)$ for all $p \in \mathcal{P}$. The following is immediate from Theorems 12 and 14.

Theorem 15: Let $\mathcal{M}=\left(s s^{\prime} \mathcal{P} \mathcal{B}, \rho\right)$ be a share minimal $(k, n)$ threshold scheme with $k<n$ that can be updated to $\mathcal{U}^{T}$. Then, for any $\Gamma^{\prime} \in \mathcal{U}^{T}$, where $\Gamma^{\prime}$ is a $\left(k^{\prime}, n^{\prime}\right)$-threshold structure

$$
H\left(b_{\Gamma^{\prime}}\right) \geq \begin{cases}\left(\min \left(n-1, n^{\prime}\right)-k^{\prime}+1\right) H(s), & \text { if } \Gamma^{\prime} \in \mathcal{U}^{+} \\ \left(n^{\prime}-k^{\prime}+1\right) H(s), & \text { if } \Gamma^{\prime} \in \mathcal{U}_{1}^{-} \\ \left(\min \left(k, n^{\prime}\right)-k^{\prime}+1\right) H(s), & \text { if } \Gamma^{\prime} \in \mathcal{U}_{2}^{-}\end{cases}
$$

We show in Section VIII that this lower bound is tight by providing a scheme that meets it.

\section{B. Updating to Smaller Regions}

We have already established results for updating to the regions $\mathcal{U}^{+}, \mathcal{U}^{-}$, and $\mathcal{U}^{T}$. Recall that, as remarked in Section III-B, it is possible that these bounds can be reduced if we only want to update to smaller regions.

The main problem with studying smaller regions is simply that it is not obvious which smaller regions might be of interest in genuine applications. Table I gives some examples of "sensible" smaller regions $\mathcal{U}$. In general, they show that so long as the update region is reasonably large then it is not possible to have a share minimal $(k, n)$-threshold scheme that can be updated to $\mathcal{U}$ using a smaller broadcast message than the bound of Theorem 15. The proofs of most of these bounds are not provided here as they can either be derived from the proofs of results in previous sections, or can be derived in a similar manner.

A few of these cases, however, do deserve special mention. Case 1 corresponds to the sequential disenrollment schemes studied in [2], [4], [15]. In this case, considering only one disenrollment, because only one participant will ever be removed from the scheme it is possible to have a small broadcast size. Case $5\left(\mathcal{U}=\mathcal{U}_{2}^{-}\right)$and Case 6 are of interest because the conditions for Lemmas 7 and 9 do not apply. In these cases, it is possible to design schemes for updating to $\mathcal{U}$ where $s=s^{\prime}$ and $H(p)=H(s)$ for all $p \in \mathcal{P}^{\prime}$. The broadcast bound indicated in Table I is only one less than that of Theorem 15 , and only when $k<n^{\prime}$. These cases, and proofs of their broadcast bounds, are given in [1] (see also Section VI-A).

\section{CONSTRUCTIONS}

We now demonstrate that the bound on the broadcast size of Theorem 15 (and thus also the bounds of Theorems 12 and 14) is tight by constructing a share-minimal $(k, n)$-threshold scheme with $k<n$ that can be updated to $\mathcal{U}^{T}$ with the minimal broadcast size indicated by Theorem 15 .

We first indicate the idea behind the construction by demonstrating an extended version of the Shamir threshold scheme (recall that Example 2 meets the broadcast bound in some, but not all, cases). We then present a construction based on Example 3 that meets the bound of Theorem 15 in all cases.

Construction 16: (Using Shamir's Scheme): We divide the scheme into three phases.

Initialization: The dealer issues each participant $p_{i}$ in $\mathcal{P}=\left\{p_{1}, \ldots, p_{n}\right\}$ with a share in each of two Shamir threshold schemes, both defined on $\mathcal{P}$ and $n-1$ "imaginary" participants 
$f_{1}, \ldots, f_{n-1}$. Scheme I is a $(k, 2 n-1)$-threshold scheme with secret $s$ and Scheme II is an $(n, 2 n-1)$-threshold scheme with secret $s^{\prime}$. Let $a(x)$ and $a^{\prime}(x)$ be the polynomials corresponding to Scheme I and Scheme II, respectively. The dealer generates distinct nonzero values $x_{1}, \ldots, x_{n}, y_{1}, \ldots, y_{n-1}$ in $\mathcal{Z}_{p}$ and publishes these. Each participant $p_{i}$ is given share $\left(a\left(x_{i}\right), a^{\prime}\left(x_{i}\right)\right)$.

Before update: Participants can use their shares of Scheme I to realize a $(k, n)$-threshold scheme.

Update: Suppose that we wish the scheme to be updated to $\Gamma^{\prime} \in \mathcal{U}^{T}$, a $\left(k^{\prime}, n^{\prime}\right)$-threshold structure on $\mathcal{P}^{\prime}=\left\{p_{1}, \ldots, p_{n^{\prime}}\right\}$. That is, we want to disenrol participants $p_{n^{\prime}+1}, \ldots, p_{n}$ and change the threshold parameter to $k^{\prime}$. In order to activate $\Gamma^{\prime}$, the dealer has two options.

1) The dealer indicates that participants should switch to using their shares in Scheme II and broadcasts

$$
a^{\prime}\left(x_{n^{\prime}+1}\right), \ldots, a^{\prime}\left(x_{n}\right), a^{\prime}\left(y_{1}\right), \ldots, a^{\prime}\left(y_{n^{\prime}-k^{\prime}}\right) .
$$

Any $k^{\prime}$ participants in $\mathcal{P}^{\prime}$ who pool their shares with the $n-k^{\prime}$ broadcast shares know $n$ points on polynomial $a^{\prime}(x)$ of degree $n-1$, so can uniquely determine $a^{\prime}(x)$ and obtain $s^{\prime}=a^{\prime}(0)$. However, any $k^{\prime}-1$ participants in $\mathcal{P}^{\prime}$ knowing the broadcast shares only have $n-1$ points on $a^{\prime}(x)$ and so obtain no information about $s^{\prime}$. The result of the broadcast is thus a $\left(k^{\prime}, n^{\prime}\right)$-threshold scheme on $\mathcal{P}^{\prime}$. The broadcast has size $H\left(b_{\Gamma^{\prime}}\right)=\left(n-k^{\prime}\right) H(s)$, which only meets the bound of Theorem 15 in a few cases (for example, when $n^{\prime} \geq n-1$ and $k^{\prime} \geq k$ ).

2) In the special case that $\Gamma^{\prime} \in \mathcal{U}_{2}^{-}$, the dealer broadcasts the values $a^{\prime}(0)-a(0)$ and

$$
a\left(x_{n^{\prime}+1}\right), \ldots, a\left(x_{n}\right), a\left(y_{1}\right), \ldots, a\left(y_{\left(k-k^{\prime}\right)-\left(n-n^{\prime}\right)}\right) .
$$

Any $k^{\prime}$ participants in $\mathcal{P}^{\prime}$ who pool their shares with the $k-k^{\prime}$ broadcast shares know $k$ points on polynomial $a(x)$ of degree $k-1$, so can determine $s=a(0)$ and hence, also knowing $a^{\prime}(0)-a(0)$, can determine $a^{\prime}(0)$. Similarly, any $k^{\prime}-1$ participants obtain no information about $s^{\prime}$. In this case, the broadcast has size $\left(k-k^{\prime}+1\right) H(s)$, which meets the bound in Theorem 15 only if $k \leq n^{\prime}$.

Construction 16 only meets the bound of Theorem 15 for some parameters. We now give a general geometric construction based on Example 3 that meets the bound in all cases.

Construction 17: (Using a Geometrical Scheme): The scheme is divided into three phases.

Initialization: The dealer issues each participant $p_{i}$ in $\mathcal{P}=$ $\left\{p_{1}, \ldots, p_{n}\right\}$ with a share in each of two geometric threshold schemes, defined as follows.

1) Let $F=\left\{f_{1}, \ldots, f_{k-1}\right\}$ be a set of "imaginary" participants. Denote $\Sigma=\operatorname{PG}(k-1, q)$ and let $\sigma: s \mathcal{P} F \rightarrow \Sigma$ be a geometric $(k, n+k-1)$-threshold scheme.

2) Let $H=\left\{h_{1}, \ldots, h_{n-1}\right\}$ be a set of "imaginary" participants. Denote $\Sigma^{\prime}=\operatorname{PG}(n-1, q)$ and let $\sigma^{\prime}: s^{\prime} \mathcal{P} H \rightarrow \Sigma^{\prime}$ be a geometric $(n, 2 n-1)$-threshold scheme.

The two shares held by each participant can be represented as a single subspace by embedding $\Sigma$ and $\Sigma^{\prime}$ as disjoint subspaces in
$\Theta=\mathrm{PG}(k+n-1, q)$ and considering the share of participant $p \in \mathcal{P}$ to be the subspace $\left\langle p^{\sigma}, p^{\sigma^{\prime}}\right\rangle$ of $\Theta$.

Before update: Participants can use their shares of $\sigma$ to realize a $(k, n)$-threshold scheme.

Update: Suppose that we wish the scheme to be updated to $\Gamma^{\prime} \in \mathcal{U}^{T}$, a $\left(k^{\prime}, n^{\prime}\right)$-threshold structure on $\mathcal{P}^{\prime}=\left\{p_{1}, \ldots, p_{n^{\prime}}\right\}$ In order to activate $\Gamma^{\prime}$, the dealer has two options.

1) Define subspaces $C^{\prime}=\left\langle\left(s^{\prime} \mathcal{P}^{\prime}\right) \sigma^{\prime}\right\rangle$ and

$$
D^{\prime}=\left\langle\left(\mathcal{P} \backslash \mathcal{P}^{\prime}\right)^{\sigma^{\prime}}, h_{1}^{\sigma^{\prime}}, \ldots, h_{n^{\prime}-k^{\prime}}^{\sigma^{\prime}}\right\rangle
$$

of $\Sigma^{\prime}$. In order to activate $\Gamma^{\prime}$, the dealer broadcasts the subspace $B^{\prime}=C^{\prime} \cap D^{\prime}$ by choosing a suitable set of $\left(\operatorname{dim} B^{\prime}+1\right)$ points of $B^{\prime}$. As $\left\langle C^{\prime}, D^{\prime}\right\rangle=\Sigma^{\prime}$, we have

$$
\begin{aligned}
\operatorname{dim} B^{\prime} & =\operatorname{dim} C^{\prime}+\operatorname{dim} D^{\prime}-\operatorname{dim}\left\langle C^{\prime}, D^{\prime}\right\rangle \\
& =\min \left(n^{\prime}, n-1\right)+\left(n-k^{\prime}-1\right)-(n-1) \\
& =\min \left(n^{\prime}, n-1\right)-k^{\prime} .
\end{aligned}
$$

If a set $K$ of $k^{\prime}$ participants in $\mathcal{P}^{\prime}$ pool their shares, then $K^{\sigma^{\prime}}$ is a subspace of dimension $k^{\prime}-1$ of $C^{\prime}$ which, by the properties of a normal rational curve, is disjoint from $D^{\prime}$ and hence $B^{\prime}$. Thus, $B^{\prime}$ and $K^{\sigma^{\prime}}$ together span $C^{\prime}$, which contains $s^{\prime \sigma^{\prime}}$, so the participants in $K$ can obtain the secret $s^{\prime}$.

For a set $L$ of $k^{\prime}-1$ participants we consider the set $X=\left\langle L^{\sigma^{\prime}}, D^{\prime}\right\rangle$. As $B^{\prime} \subseteq D^{\prime}$, it follows that $B^{\prime} \subseteq X$. The set $X$ is generated by points of the normal rational curve, hence,

$$
\operatorname{dim} X=\left(k^{\prime}-1\right)+\left(\left(n-n^{\prime}\right)+\left(n^{\prime}-k^{\prime}\right)\right)-1=n-2 .
$$

By the properties of a normal rational curve, $X$ does not contain any further point of the normal rational curve, so in particular, does not contain $s^{\prime}$. This implies that a maximal unauthorized $\left(k^{\prime}-1\right)$-set $L \subseteq \mathcal{P}^{\prime}$ together with the participants $\mathcal{P} \backslash \mathcal{P}^{\prime}$ and broadcast $B^{\prime}$ cannot obtain $s^{\prime}$. The result of the broadcast is thus a $\left(k^{\prime}, n^{\prime}\right)$-threshold scheme on $\mathcal{P}^{\prime}$. We refer to this construction process as updating with $\sigma^{\prime}$. If $n^{\prime}=n$ then $H\left(b_{\Gamma^{\prime}}\right)=\left(n-k^{\prime}\right) H(s)$. If $n^{\prime}<n$ then $H\left(b_{\Gamma^{\prime}}\right)=\left(n^{\prime}-k^{\prime}+1\right) H(s)$. So, updating with $\sigma^{\prime}$ achieves the bound of Theorem 15 in the following cases: a) $\Gamma^{\prime} \in \mathcal{U}^{+} \cup \mathcal{U}_{1}^{-}$; b) $\Gamma^{\prime} \in \mathcal{U}_{2}^{-}$and $n^{\prime}<k$.

2) For the remaining case, that is, $\Gamma^{\prime} \in \mathcal{U}_{2}^{-}$and $k \leq n^{\prime}$, we describe a similar process referred to as updating with $\sigma$. Although we show this for all $\Gamma^{\prime} \in \mathcal{U}_{2}^{-}$, it is only optimal for $k \leq n^{\prime}$. Define subspaces $C=\left\langle\left(s \mathcal{P}^{\prime}\right)^{\sigma}\right\rangle$ and

$$
D=\left\langle\left(\mathcal{P} \backslash \mathcal{P}^{\prime}\right)^{\sigma}, f_{1}^{\sigma}, \ldots, f_{\left(k-k^{\prime}\right)-\left(n-n^{\prime}\right)}^{\sigma}\right\rangle
$$

of $\Sigma$. In order to activate $\Gamma^{\prime}$, the dealer broadcasts the subspace $B=C \cap D$, where

$$
\begin{aligned}
\operatorname{dim} B & =\operatorname{dim} C+\operatorname{dim} D-\operatorname{dim}\langle C, D\rangle \\
& =\min \left(n^{\prime}, k-1\right)+\left(k-k^{\prime}-1\right)-(k-1) \\
& =\min \left(n^{\prime}, k-1\right)-k^{\prime}
\end{aligned}
$$

and also a point $W$ on the line $\left\langle s^{\sigma}, s^{\prime \sigma^{\prime}}\right\rangle$ in $\Sigma^{T}$, where $W \notin\left\{s^{\sigma}, s^{\prime \sigma^{\prime}}\right\}$. This gives a $\left(k^{\prime}, n^{\prime}\right)$-threshold scheme on $\mathcal{P}^{\prime}$ with secret $s^{\prime}$ and with broadcast satisfying

$H\left(b_{\Gamma^{\prime}}\right)=(\operatorname{dim} B+1)+1=\min \left(n^{\prime}, k-1\right)-k^{\prime}+2$. Thus, for the case $\Gamma^{\prime} \in \mathcal{U}_{2}^{-}$and $k \leq n^{\prime}$, updating with $\sigma$ meets the bound of Theorem 15 . 
Hence, we have shown that the bound in Theorem 15 can always be met by either updating with $\sigma^{\prime}$ or updating with $\sigma$. Note also that if we wanted to make the assumption that the participants cannot determine $s^{\prime}$ before the broadcast then we would need $\sigma^{\prime}$ to be an $(n+1,2 n)$-threshold scheme, in which case we would have

$$
H\left(b_{\Gamma^{\prime}}\right)= \begin{cases}\left(k-k^{\prime}+1\right) H(s), & \text { if } \Gamma^{\prime} \in \mathcal{U}_{2}^{-} \text {and } k<n^{\prime} \\ \left(n^{\prime}-k^{\prime}+1\right) H(s), & \text { otherwise. }\end{cases}
$$

We now give an example to illustrate and compare Constructions 16 and 17.

Example 18: Suppose we start with $\Gamma$, a $(3,5)$-threshold scheme on $\mathcal{P}^{\prime}=\left\{p_{1}, p_{2}, p_{3}, p_{4}, p_{5}\right\}$, and we want to update to $\Gamma^{\prime}$, a $(2,3)$-threshold scheme on $\mathcal{P}^{\prime}=\left\{p_{1}, p_{2}, p_{3}\right\}$. We will show how to update using both Constructions 16 and 17 and show that Construction 17 results in a smaller broadcast size than Construction 16. Note that $\Gamma^{\prime}$ is in the region $\mathcal{U}_{1}^{-}$and so we will use the first update option described in each construction.

We will work over $\operatorname{GF}(p)$, $p$ a large odd prime. We begin with the polynomial Construction 16. Let $\mathcal{F}=\left\{f_{1}, \ldots, f_{4}\right\}$ and note that Scheme I is a $(3,9)$-threshold scheme on $\mathcal{P} \cup \mathcal{F}$ and Scheme II is a $(5,9)$-threshold scheme on $\mathcal{P} \cup \mathcal{F}$. Suppose Scheme I has associated polynomial $a(x)$ and Scheme II has associated polynomial

$$
a^{\prime}(x)=a_{0}^{\prime}+a_{1}^{\prime} x+a_{2}^{\prime} x^{2}+a_{3}^{\prime} x^{3}+a_{4}^{\prime} x^{4} .
$$

Suppose the public values for $p_{1}, \ldots, p_{5}$ are $x_{1}=1, x_{2}=$ $-1, x_{3}=2, x_{4}=-2$, and $x_{5}=3$, and so their shares are $\left(a\left(x_{1}\right), a^{\prime}\left(x_{1}\right)\right), \ldots,\left(a\left(x_{5}\right), a^{\prime}\left(x_{5}\right)\right)$, respectively. To perform the update to $\Gamma^{\prime}$ as in Construction 16, we need to broadcast three pieces of information, namely, $a^{\prime}\left(x_{4}\right), a^{\prime}\left(x_{5}\right)$, and $a^{\prime}\left(y_{1}\right)$. This would enable an authorized set in $\Gamma^{\prime}$ to obtain $s^{\prime}=a_{0}^{\prime}$ by completely determining all the coefficients $a_{0}^{\prime}, \ldots, a_{4}^{\prime}$ of $a^{\prime}(x)$.

We can think of this polynomial Scheme II as a geometric construction in $\operatorname{PG}(4, q)$ as follows. We can represent $p_{i}$ 's information in Scheme II as

$$
\left[\begin{array}{lllll}
1 & x_{i} & x_{i}^{2} & x_{i}^{3} & x_{i}^{4}
\end{array}\right]\left[\begin{array}{lllll}
a_{0}^{\prime} & a_{1}^{\prime} & a_{2}^{\prime} & a_{3}^{\prime} & a_{4}^{\prime}
\end{array}\right]^{t}=a^{\prime}\left(x_{i}\right) .
$$

In the geometric interpretation, we think of $\left(1, x_{i}, x_{i}^{2}, x_{i}^{3}, x_{i}^{4}\right)$ as a point in $\operatorname{PG}(4, q)$ associated with participant $p_{i}$. Thus, each participant is associated with a point on a normal rational curve as described in Example 3. (A similar interpretation applies for Scheme I.)

Thus, by using this geometric interpretation, we can illustrate how to update from the polynomial $a^{\prime}(x)$ to $\Gamma^{\prime}$ using Construction 17. Using the same notation as in the construction, let $\sigma^{\prime}$ be a geometric $(5,9)$-threshold scheme on $\mathcal{P} \cup\left\{h_{1}, \ldots, h_{4}\right\}$ (such that $\sigma^{\prime}$ corresponds to the polynomial $a^{\prime}(x)$ using the above interpretation). Let $C^{\prime}=\left\langle\left(s^{\prime} \mathcal{P}^{\prime}\right)^{\sigma^{\prime}}\right\rangle$. This, subspace is represented by the matrix equation

$$
C_{1} \alpha=\beta
$$

where

$$
C_{1}=\left[\begin{array}{ccccc}
1 & 0 & 0 & 0 & 0 \\
1 & 1 & 1 & 1 & 1 \\
1 & -1 & 1 & -1 & 1 \\
1 & 2 & 4 & 8 & 16
\end{array}\right], \alpha=\left[\begin{array}{c}
a_{0}^{\prime} \\
a_{1}^{\prime} \\
a_{2}^{\prime} \\
a_{3}^{\prime} \\
a_{4}^{\prime}
\end{array}\right], \beta=\left[\begin{array}{c}
a_{0}^{\prime} \\
a^{\prime}\left(x_{1}\right) \\
a^{\prime}\left(x_{2}\right) \\
a^{\prime}\left(x_{3}\right)
\end{array}\right] .
$$

We note that the connection between the polynomial scheme and the geometrical scheme is through the matrix $C_{1}$. In the polynomial scheme, if we know $C_{1} \alpha=\beta$, we know all linear combinations of the equations represented by $C_{1} \alpha=\beta$. In the geometric interpretation, this corresponds to the following: if we know the points represented by $C_{1}$, then we know all the linear combinations of these points, that is, we know the subspace generated by these points. Thus, the geometrical scheme is a straightforward generalization of the polynomial scheme. Note that in the polynomial scheme, broadcast information is limited to points on the polynomial. However, in the geometric scheme, the broadcast information is more flexible (which is why we can obtain smaller broadcasts). In the geometric scheme, broadcast information is not limited to points on the normal rational curve, we can broadcast any point in $\operatorname{PG}(4, q)$. To broadcast the point $\left(t_{1}, t_{2}, t_{3}, t_{4}, t_{5}\right) \in \operatorname{PG}(4, q)$, we broadcast the value $\left[t_{1}, t_{2}, t_{3}, t_{4}, t_{5}\right] \alpha$, that is, we can broadcast any linear combination of the coefficients of $a^{\prime}(x)$.

We now use this geometric setup to show how Construction 17 performs the required update. We need one imaginary participant $h_{1}$; suppose $h_{1}$ has public value $y_{1}=-3$. We calculate $D^{\prime}=\left\langle\left(p_{4} p_{5} h_{1}\right)^{\sigma^{\prime}}\right\rangle$, with matrix equation $D_{1} \alpha=\beta^{\prime}$, where

$D_{1}=\left[\begin{array}{ccccc}1 & -2 & 4 & -8 & 16 \\ 1 & 3 & 9 & 27 & 81 \\ 1 & -3 & 9 & -27 & 81\end{array}\right], \alpha=\left[\begin{array}{l}a_{0}^{\prime} \\ a_{1}^{\prime} \\ a_{2}^{\prime} \\ a_{3}^{\prime} \\ a_{4}^{\prime}\end{array}\right], \beta=\left[\begin{array}{l}a^{\prime}\left(x_{4}\right) \\ a^{\prime}\left(x_{5}\right) \\ a^{\prime}\left(y_{1}\right)\end{array}\right]$.

Row reducing $D_{1}$ yields

$$
\left[\begin{array}{ccccc}
1 & 0 & 0 & 18 & -36 \\
0 & 1 & 0 & 9 & 0 \\
0 & 0 & 1 & -2 & 13
\end{array}\right]
$$

and so a general point $R$ of $D^{\prime}$ has form

$$
(a, b, c, 18 a+9 b-2 c,-36 a+13 c) .
$$

To find where $C^{\prime}$ intersects $D^{\prime}$ we first row reduce $C_{1}$ to obtain

$$
\left[\begin{array}{ccccc}
1 & 0 & 0 & 0 & 0 \\
0 & 1 & 0 & 0 & -2 \\
0 & 0 & 1 & 0 & 1 \\
0 & 0 & 0 & 1 & 2
\end{array}\right] .
$$

So the general point $R$ of $D^{\prime}$ is in $C^{\prime}$ if and only if it is a linear combination of the rows of $C_{1}$, that is,

$$
\begin{aligned}
R & =(a, b, c, 18 a+9 b-2 c,(-2 b)+(c)+2(18 a+9 b-2 c)) \\
& =(a, b, c, 18 a+9 b-2 c, 36 a+16 b-3 c) .
\end{aligned}
$$

So $R \in C^{\prime} \cap D^{\prime}$ if and only if $-36 a+13 c=36 a-16 b-3 c$, that is, $c=9 / 2 a+b$. Hence, $R=a(2,0,9,18,45)+$ $b(0,2,2,12,26)$. Thus, the broadcast information is the two values

$$
b_{1}=[2,0,9,18,45] \alpha, \quad b_{2}=[0,2,2,12,26] \alpha .
$$

We now verify that authorized sets can obtain the secret $s^{\prime}=a_{0}^{\prime}$. The authorized set $\left\{p_{1}, p_{2}\right\}$ knows the following matrix equation whose four rows correspond to $p_{1}$ and $p_{2}$ 's shares, and the broadcast values $b_{1}$ and $b_{2}$

$$
\left[\begin{array}{ccccc}
1 & 1 & 1 & 1 & 1 \\
1 & -1 & 1 & -1 & 1 \\
2 & 0 & 9 & 18 & 45 \\
0 & 2 & 2 & 12 & 26
\end{array}\right]\left[\begin{array}{c}
a_{0}^{\prime} \\
a_{1}^{\prime} \\
a_{2}^{\prime} \\
a_{3}^{\prime} \\
a_{4}^{\prime}
\end{array}\right]=\left[\begin{array}{c}
a^{\prime}\left(x_{1}\right) \\
a^{\prime}\left(x_{2}\right) \\
b_{1} \\
b_{2}
\end{array}\right]
$$


To show that $a_{0}^{\prime}$ can be calculated, we apply row operations to

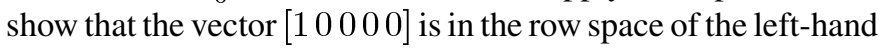
matrix. This is so since the row reduced form of that matrix is

$$
\left[\begin{array}{ccccc}
1 & 0 & 0 & 0 & 0 \\
0 & 1 & 0 & 0 & -2 \\
0 & 0 & 1 & 0 & 1 \\
0 & 0 & 0 & 1 & 2
\end{array}\right] .
$$

Similarly, for the remaining authorized sets $\left\{p_{1}, p_{3}\right\}$ and $\left\{p_{2}, p_{3}\right\}$. We do not need to check the unauthorized sets as the span of any four points on the normal rational curve does not contain any further point of the normal rational curve, so does not contain $s^{\prime}$.

Hence, using Construction 17, we only need to broadcast two pieces of information compared to three pieces for Construction 16. Note that with Construction 16, authorized sets have enough information to determine the entire polynomial $a^{\prime}(x)$. However, with Construction 17, although authorized sets have enough information to determine $s^{\prime}=a_{0}^{\prime}$, they do not necessarily have enough information to determine $a^{\prime}(x)$.

\section{CONCLUDING REMARKS}

We have established the minimal broadcast necessary to update the parameters of a share-minimal threshold scheme, and demonstrated an optimal scheme for achieving these bounds. We showed these results for achieving one parameter update. A natural question to consider is the situation where we want more than one, say two updates. If $\Gamma$ on $\mathcal{P}$ is updated to $\Gamma^{\prime}$ on $\mathcal{P}^{\prime} \subseteq \mathcal{P}$ (using $b_{\Gamma^{\prime}}$ ), then updated to $\Gamma^{\prime \prime}$ on $\mathcal{P}^{\prime \prime}$ (using $b_{\Gamma^{\prime \prime}}$ ), we would need $\mathcal{P}^{\prime \prime} \subseteq \mathcal{P}^{\prime}$. So we would need to add the extra condition

$$
H\left(s^{\prime \prime} \mid A b_{\Gamma^{\prime}} b_{\Gamma^{\prime \prime}}\right)= \begin{cases}0, & \text { if }\left|A \cap \mathcal{P}^{\prime \prime}\right| \geq k^{\prime \prime} \\ H\left(s^{\prime \prime}\right), & \text { if }\left|A \cap \mathcal{P}^{\prime \prime}\right| \leq k^{\prime \prime}-1 .\end{cases}
$$

The next step would be to investigate the independence of the secrets $s, s^{\prime}$, and $s^{\prime \prime}$. Under what circumstances would it hold that $H\left(s^{\prime \prime} \mid s s^{\prime}\right)=H\left(s^{\prime \prime}\right)$ ?

Third, for which access structures $\Gamma$ and update collections $\mathcal{U}$ (containing $\Gamma^{\prime}$ ) and $\mathcal{U}^{\prime \prime}$ (containing $\Gamma^{\prime \prime}$ ) do we need to have $H(p) \geq H(s)+H\left(s^{\prime}\right)+H\left(s^{\prime \prime}\right)$ ?

Fourth, we would expect the same bounds for $b_{\Gamma^{\prime \prime}}$ as for $b_{\Gamma^{\prime}}$ and so we could extend Construction 17 in Section VIII by having an extra $k-1$ imaginary participants in schemes $\sigma$ and $\sigma^{\prime}$, and having a third scheme $\sigma^{\prime \prime}$, a geometric $(n, 2 n-1)$ threshold scheme.

Another interesting generalization is to consider threshold schemes that are not share-minimal. In such schemes, it is possible to reduce the broadcast size at the expense of an increase in the amount of information stored as shares. As mentioned in Section I, the extreme case of this concession is to give participants one share for every possible new threshold parameter set and then simply broadcast a message indicating which new set of shares to move to in order to enable the new parameter change. The pattern of the intermediate tradeoffs between this extreme case and the share-minimal schemes discussed in this paper remains undetermined and would be worthy of further investigation.

\section{APPENDIX \\ PROOFS OF MAIN THEOREMS}

\section{A. Proof for Theorem 12}

We proceed to prove this result by induction, commencing with the case $k=1$ and then the general case.

Increasing the Threshold: The Case $k=1$ : We aim to prove the lower bound on the broadcast size for the case $k=1$. We first prove two technical lemmas and then establish the bound for this case.

Let $n>1$ and $\mathcal{M}=\left(s s^{\prime} \mathcal{P B}, \rho\right)$ be a share-minimal $(1, n)$-threshold scheme that can be updated to $\mathcal{U}^{+}(1, n)$. Let $\Gamma^{\prime} \in \mathcal{U}^{+}(1, n)$ be a $\left(k^{\prime}, n^{\prime}\right)$-threshold structure on $\mathcal{P}^{\prime} \subseteq \mathcal{P}$. By share minimality we have $H(p)=2 H(s)$ for each $p \in \mathcal{P}$. Essentially, one part of each share can be thought of as relating to the original $(1, n)$-threshold structure $\Gamma$ and the other part can be thought of as relating to the new threshold structure $\Gamma^{\prime} \in \mathcal{U}^{+}(1, n)$. To see this, we factor out the first part by letting $\sigma \in[s]$ and defining a new probability distribution $\tau$ on $\left\langle s^{\prime} \mathcal{P B}\right\rangle_{\rho}$ by $\tau=\rho_{s^{\prime}} \mathcal{P B} \mid s=\sigma$. The following lemma shows that $\left(s^{\prime} \mathcal{P B}, \tau\right)$ is "almost" a $\left(k^{\prime}, n^{\prime}\right)$-threshold scheme.

Lemma 19: Let $\sigma \in[s]$ and $A \subseteq \mathcal{P}^{\prime}$ be a $k^{\prime}$-set. Then

a) $H_{\tau}\left(s^{\prime}\right)=H\left(s^{\prime}\right)$;

b) $H_{\tau}(p)=H_{\tau}\left(s^{\prime}\right)$;

c) $H_{\tau}\left(s^{\prime} \mid A b_{\Gamma^{\prime}}\right)=0$

d) $H_{\tau}\left(s^{\prime} \mid(A \backslash p)\left(\mathcal{P} \backslash \mathcal{P}^{\prime}\right) b_{\Gamma^{\prime}}\right)=H_{\tau}\left(s^{\prime}\right)$ for any $p \in A$.

Proof: By definition we have

$$
\begin{aligned}
H\left(s^{\prime} \mid A b_{\Gamma^{\prime}}\right) & =0 \\
H\left(s^{\prime} \mid(A \backslash p) b_{\Gamma^{\prime}}\left(\mathcal{P} \backslash \mathcal{P}^{\prime}\right)\right) & =H\left(s^{\prime}\right), \quad \text { for all } p \in A .
\end{aligned}
$$

Part a) follows immediately by noting that Theorem 11 a) implies that $\rho_{s^{\prime}}=\tau_{s^{\prime}}$. Now choose $X \subseteq \mathcal{P}, X \neq \emptyset$. It follows from the definition of conditional entropy that

$$
\sum_{\sigma \in[s]} \rho_{s}(\sigma) H_{\tau}\left(s^{\prime} \mid b_{\Gamma^{\prime}} X\right)=H\left(s^{\prime} \mid b_{\Gamma^{\prime}} X s\right)=H\left(s^{\prime} \mid b_{\Gamma^{\prime}} X\right)
$$

as $|X| \geq 1$ implies $H(s \mid X)=0$.

To prove part c), let $X=A$. By (7) and (9) it follows that $H_{\tau}\left(s^{\prime} \mid b_{\Gamma^{\prime}} A\right)=0$, as required. For part d), let $p \in A$ and choose $X=(A \backslash p)\left(\mathcal{P} \backslash \mathcal{P}^{\prime}\right)$. Since $H_{\tau}\left(s^{\prime} \mid b_{\Gamma^{\prime}} X\right) \leq H_{\tau}\left(s^{\prime}\right)$ for all $\sigma \in[s]$, by (8) and (9) it follows that $H_{\tau}\left(s^{\prime} \mid b_{\Gamma^{\prime}} X\right)=$ $H_{\tau}\left(s^{\prime}\right)$, as required.

Finally, for part b), apply Lemma 10 to parts c) and d) to obtain $H_{\tau}(p) \geq H_{\tau}(s)=H\left(s^{\prime}\right)$, the equality by a). As

$$
\begin{aligned}
\sum_{\sigma \in[s]} \rho_{s}(\sigma) H_{\tau}(p) & =H(p \mid s)=H(s \mid p)+H(p)-H(s) \\
& =H(p)-H(s)=H(s)
\end{aligned}
$$

it follows that $H_{\tau}(p)=H(s)$ for all $\sigma \in[s]$.

We now prove some further properties of $\tau$ before establishing the bound.

Lemma 20: With respect to $\tau$ we have the following.

a) The variables $\{p \mid p \in \mathcal{P}\}$ are independent.

b) For $p \in \mathcal{P}$, we have $H_{\tau}\left(s^{\prime} \mid(\mathcal{P} \backslash p)\right)=H_{\tau}\left(s^{\prime}\right)$.

c) If $Y \subseteq \mathcal{P}^{\prime}$ is a $\left(k^{\prime}-1\right)$-set then $H_{\tau}\left(p \mid b_{\Gamma^{\prime}} Y s^{\prime}\right)=0$. 
Proof:

a) To show independence, we show that

$$
H_{\tau}(p \mid(\mathcal{P} \backslash p))=H_{\tau}(p) .
$$

Let $\Gamma^{\prime} \in \mathcal{U}^{+}(1, n)$ be the $(1,1)$-threshold structure on $\mathcal{P}^{\prime}=\{p\}$. From Lemma $19 \mathrm{c}$ ) and d) we have

$$
H_{\tau}\left(s^{\prime} \mid \mathcal{P} b_{\Gamma^{\prime}}\right)=0 \text { and } H_{\tau}\left(s^{\prime} \mid(\mathcal{P} \backslash p) b_{\Gamma^{\prime}}\right)=H_{\tau}\left(s^{\prime}\right) \text {. }
$$

Applying Lemma 10 we get

$$
H_{\tau}\left(s^{\prime}\right) \leq H_{\tau}\left(p \mid(\mathcal{P} \backslash p) b_{\Gamma^{\prime}}\right) \leq H_{\tau}(p) .
$$

The result follows by Lemma $19 \mathrm{~b}$ ).

b) Let $\Gamma^{\prime} \in \mathcal{U}^{+}(1, n)$ be the $(n, n)$-threshold structure. Then, for $p \in \mathcal{P}, \mathcal{P} \backslash p \notin \Gamma^{\prime}$. Thus, by Lemma $19 \mathrm{~d}$ )

$$
H_{\tau}\left(s^{\prime} \mid(\mathcal{P} \backslash p) b_{\Gamma^{\prime}}\right)=H_{\tau}\left(s^{\prime}\right)
$$

and so

$$
H_{\tau}\left(s^{\prime} \mid(\mathcal{P} \backslash p)\right)=H_{\tau}\left(s^{\prime}\right) .
$$

c) Using the results of Section II-A,

$$
\begin{aligned}
& 0 \leq H_{\tau}\left(p \mid b_{\Gamma^{\prime}} Y s^{\prime}\right) \\
& =H_{\tau}\left(b_{\Gamma^{\prime}} p Y s^{\prime}\right)-H_{\tau}\left(b_{\Gamma^{\prime}} Y s^{\prime}\right) \\
& \left.=H_{\tau}\left(b_{\Gamma^{\prime}} p Y\right)-H_{\tau}\left(b_{\Gamma^{\prime}} Y s^{\prime}\right), \quad \text { by Lemma } 19 \mathrm{c}\right) \\
& =H_{\tau}\left(b_{\Gamma^{\prime}} p Y\right)-H_{\tau}\left(b_{\Gamma^{\prime}} Y\right)-H_{\tau}\left(s^{\prime}\right) \text {, } \\
& \text { by Lemma } 19 \text { d) } \\
& =H_{\tau}\left(p \mid b_{\Gamma^{\prime}} Y\right)-H_{\tau}\left(s^{\prime}\right) \\
& \leq H_{\tau}(p)-H_{\tau}\left(s^{\prime}\right) \\
& =0 \text {, } \\
& \text { by Lemma } 19 \text { b). }
\end{aligned}
$$

Hence equality holds throughout.

We can now prove the lower bound on the size of broadcast for the case $k=1$.

Lemma 21: Let $\mathcal{M}=\left(s s^{\prime} \mathcal{P B}, \rho\right)$ be a share minimal $(1, n)$ threshold scheme with $1<n$ that can be updated to $\mathcal{U}^{+}(1, n)$. Then, for any $\Gamma^{\prime} \in \mathcal{U}^{+}(1, n)$ where $\Gamma^{\prime}$ is a $\left(k^{\prime}, n^{\prime}\right)$-threshold structure

$$
H\left(b_{\Gamma^{\prime}}\right) \geq \begin{cases}\left(n^{\prime}-k^{\prime}+1\right) H(s), & \text { if } n^{\prime}<n \\ \left(n^{\prime}-k^{\prime}\right) H(s), & \text { if } n^{\prime}=n .\end{cases}
$$

Proof: Let $\Gamma^{\prime} \in \mathcal{U}^{+}(1, n)$ be a $\left(k^{\prime}, n^{\prime}\right)$-threshold structure defined on $\mathcal{P}^{\prime}=\left\{p_{1}, \ldots, p_{n^{\prime}}\right\}$.

$$
\begin{aligned}
H_{\tau}\left(b_{\Gamma^{\prime}}\right) \geq & \mathrm{I}_{\tau}\left(b_{\Gamma^{\prime}} ; p_{1}, \ldots, p_{n^{\prime}} \mid s^{\prime}\right) \text { by }(5) \\
= & H_{\tau}\left(p_{1}, \ldots, p_{n^{\prime}} \mid s^{\prime}\right)-H_{\tau}\left(p_{1}, \ldots, p_{n^{\prime}} \mid b_{\Gamma^{\prime}} s^{\prime}\right) \\
= & H_{\tau}\left(p_{1}, \ldots, p_{n^{\prime}} \mid s^{\prime}\right) \\
& -\sum_{i=1}^{n^{\prime}} H_{\tau}\left(p_{i} \mid b_{\Gamma^{\prime}} p_{1}, \ldots, p_{(i-1)} s^{\prime}\right) .
\end{aligned}
$$

For $1 \leq i \leq k^{\prime}-1$ we have

$$
H_{\tau}\left(p_{i} \mid b_{\Gamma^{\prime}} p_{1}, \ldots, p_{(i-1)} s^{\prime}\right) \leq H_{\tau}\left(p_{i}\right)=H_{\tau}\left(s^{\prime}\right)
$$

by Lemma 19 b). For $k^{\prime} \leq i \leq n^{\prime}$ we have

$$
H_{\tau}\left(p_{i} \mid b_{\Gamma^{\prime}} p_{1}, \ldots, p_{(i-1)} s^{\prime}\right)=0
$$

by Lemma $20 \mathrm{c})$. So

$$
H_{\tau}\left(b_{\Gamma^{\prime}}\right) \geq H_{\tau}\left(p_{1}, \ldots, p_{n^{\prime}} \mid s^{\prime}\right)-\left(k^{\prime}-1\right) H_{\tau}\left(s^{\prime}\right) .
$$

If $n^{\prime}=n$, then by Lemma 20 a) the $p_{i}$ are independent, and so

$$
\begin{aligned}
H_{\tau}\left(p_{1}, \ldots, p_{n^{\prime}} \mid s^{\prime}\right)= & H_{\tau}\left(p_{1}, \ldots, p_{n^{\prime}-1} \mid s^{\prime}\right) \\
& +H_{\tau}\left(p_{n^{\prime}} \mid p_{1}, \ldots, p_{n^{\prime}-1}\right) \\
\geq & H_{\tau}\left(p_{1}, \ldots, p_{n^{\prime}-1} \mid s^{\prime}\right) \\
= & H_{\tau}\left(p_{1}, \ldots, p_{n^{\prime}-1}\right)
\end{aligned}
$$

by Lemma 20 b). Hence, by Lemma 20 a)

$$
H_{\tau}\left(p_{1}, \ldots, p_{n^{\prime}} \mid s^{\prime}\right)=\left(n^{\prime}-1\right) H_{\tau}\left(s^{\prime}\right) .
$$

If $n^{\prime}<n$, then by Lemma 20 we have

$$
\begin{aligned}
H_{\tau}\left(p_{1}, \ldots, p_{n^{\prime}} \mid s^{\prime}\right) & =H_{\tau}\left(p_{1}, \ldots, p_{n^{\prime}}\right), & & \text { by Lemma } 20 \mathrm{~b}) \\
& =n^{\prime} H_{\tau}\left(s^{\prime}\right), & & \text { by Lemma } 20 \mathrm{a}) .
\end{aligned}
$$

Thus, in both cases we have $H_{\tau}\left(p_{1}, \ldots, p_{n^{\prime}} \mid s^{\prime}\right) \geq n^{\prime} H_{\tau}\left(s^{\prime}\right)$. Hence, combining the cases and using Lemma 19 a) and (1) we get

$$
H_{\tau}\left(b_{\Gamma^{\prime}}\right) \geq \begin{cases}\left(n^{\prime}-k^{\prime}+1\right) H(s), & \text { if } n^{\prime}<n \\ \left(n^{\prime}-k^{\prime}\right) H(s), & \text { if } n^{\prime}=n .\end{cases}
$$

As $H\left(b_{\Gamma^{\prime}}\right)=\sum_{\sigma \in[s]} H_{\tau}\left(b_{\Gamma^{\prime}}\right)$, the theorem follows, as required.

Increasing the Threshold: The General Case: We are now ready to prove Theorem 12 by induction on $k$.

If $k=1$ then the result is proved by Lemma 21. Suppose $k>1$. Let $K \subseteq \mathcal{P}$ be a $(k-1)$-set and let $\mathcal{Q}=\mathcal{P} \backslash K$. Let $\kappa \in[K]_{\rho}$ and let the probability distribution $\mu$ on $\left\langle s s^{\prime} \mathcal{Q B}\right\rangle_{\rho}$ be defined by $\mu=\rho_{s s^{\prime} \mathcal{Q B} \mid K=\kappa}$. As $\rho_{K}(\kappa)>0$, it follows that $\left[s s^{\prime} \mathcal{Q B}\right]_{\mu} \neq \emptyset$.

Let $\mathcal{N}=\left(s s^{\prime} \mathcal{Q B}, \mu\right)$ be the scheme corresponding to $\mu$. In order to apply Lemma 21 , we now show that $\mathcal{N}$ is a $(1, n-(k-1))$ threshold scheme that can be updated to $\mathcal{U}^{+}(1, n-(k-1))$, for an appropriate choice of broadcast.

Let $p \in \mathcal{Q}$. Now $H(s \mid p K)=0$ so

$$
0=H(s \mid p(K=\kappa))=H_{\mu}(s \mid p) .
$$

as required. Since $|K|<k, H(s \mid K=\kappa)=H(s)$ and so $H_{\mu}(s)=H(s)$. Part (A) of Definition 6 is thus satisfied.

Let $\Pi \in \mathcal{U}^{+}(1, n-(k-1))$ be an $(l, m)$-threshold access structure on $\mathcal{Q}^{\prime} \subseteq \mathcal{Q}$. Let $\Gamma^{\prime}$ be the $\left(k^{\prime}, n^{\prime}\right)$-threshold structure on $\mathcal{P}^{\prime}=K \mathcal{Q}^{\prime}$ with $k^{\prime}=l+(k-1)$ and $n^{\prime}=m+(k-1)$. We show that $b_{\Gamma^{\prime}}$ is the broadcast variable for $\Pi$ in $\mu$. Let $A \subseteq \mathcal{Q}^{\prime}$ be an $l$-set, so $|A K|=k^{\prime}$. Since $\mathcal{M}$ can be updated to $\Gamma^{\prime}$, $H\left(s^{\prime} \mid A K b_{\Gamma^{\prime}}\right)=0$. This implies that $H\left(s^{\prime} \mid A(K=\kappa) b_{\Gamma^{\prime}}\right)=0$ and so $H_{\mu}\left(s^{\prime} \mid A b_{\Gamma^{\prime}}\right)=0$. Now let $p \in A$. Since

$$
H\left(s^{\prime} \mid(A \backslash p) K\left(\mathcal{P} \backslash \mathcal{P}^{\prime}\right) b_{\Gamma^{\prime}}\right)=H\left(s^{\prime}\right)
$$

it follows from Section II-A that

$$
H_{\mu}\left(s^{\prime} \mid(A \backslash p)\left(\mathcal{P} \backslash \mathcal{P}^{\prime}\right) b_{\Gamma^{\prime}}\right)=H\left(s^{\prime}\right) .
$$

Since $|K|<k^{\prime}, H\left(s^{\prime} \mid K\right)=H\left(s^{\prime}\right)$ and so $H_{\mu}\left(s^{\prime}\right)=H\left(s^{\prime}\right)$. Part (B) of Definition 6 is thus also satisfied.

So we have $\mathcal{N}$ a $(1, n-(k-1))$-threshold scheme which can be updated to $\Pi \in \mathcal{U}^{+}(1, n-(k-1))$ a $(l, m)$-threshold access 
structure using broadcast variable $b_{\Gamma^{\prime}}$. We apply Lemma 21 to obtain

$$
\begin{aligned}
& H_{\mu}\left(b_{\Gamma^{\prime}}\right) \geq \begin{cases}(m-l+1) H_{\mu}(s), & \text { if } m<n-(k-1) \\
(m-l) H_{\mu}(s), & \text { if } m=n-(k-1)\end{cases} \\
&= \begin{cases}\left(n^{\prime}-k^{\prime}+1\right) H_{\mu}(s), & \text { if } n^{\prime}<n \\
\left(n^{\prime}-k^{\prime}\right) H_{\mu}(s), & \text { if } n^{\prime}=n .\end{cases}
\end{aligned}
$$

Since we have already shown that $H(s)=H_{\mu}(s)$, and since

$$
\begin{aligned}
\sum_{\kappa \in[K]} \rho_{K}(\kappa) H_{\mu}\left(b_{\Gamma^{\prime}}\right) & =\sum_{\kappa \in[K]} \rho_{K}(\kappa) H\left(b_{\Gamma^{\prime}} \mid K=\kappa\right) \\
& =H\left(b_{\Gamma^{\prime}} \mid K\right) \\
& \leq H\left(b_{\Gamma^{\prime}}\right)
\end{aligned}
$$

and Theorem 12 is proved.

Finally, we note that if the model is restricted to include the extra requirement that the participants belonging to $\Gamma^{\prime}$ cannot obtain $s^{\prime}$ prior to knowledge of the broadcast $b_{\Gamma^{\prime}}$ (in other words, $\left.H_{\tau}\left(s^{\prime} \mid \mathcal{P}\right)=H_{\tau}\left(s^{\prime}\right)\right)$ then it follows that

$$
H\left(p_{1}, \ldots, p_{n^{\prime}} \mid s^{\prime}\right)=n^{\prime} H(s)
$$

and from (10) we get the simplified bound of

$$
H\left(b_{\Gamma^{\prime}}\right) \geq\left(n^{\prime}-k^{\prime}+1\right) H(s)
$$

instead of the bound in Theorem 12 .

\section{B. Proof for Theorem 14}

We have already seen that the two regions $\mathcal{U}_{1}^{-}$and $\mathcal{U}_{2}^{-}$are in some respects fundamentally different. In fact, to prove a lower bound on the broadcast size for updating to $\mathcal{U}^{-}$we need two different approaches for each of these two regions. We prove these results separately in Theorems 23 and 24. The bound in Theorem 14 then follows immediately.

Decreasing the Threshold: Updating to $\mathcal{U}_{1}^{-}$: Recall that our aim is to establish a bound for updating to $\mathcal{U}^{-}$. In this subsection, we consider schemes for updating to $\mathcal{U}^{-}$but will only be concerned with how big the broadcast size is in the case that the new threshold parameters belong to $\mathcal{U}_{1}^{-}$. We proceed by induction on the new threshold parameter, first proving the bound for updating to $k^{\prime}=1$ and then for updating to general $k^{\prime}$.

Lemma 22: Let $\mathcal{M}=\left(s s^{\prime} \mathcal{P} \mathcal{B}, \rho\right)$ be a share minimal $(k, n)$ threshold scheme with $k<n$ that can be updated to $\mathcal{U}^{-}$. Then for any $\left(1, n^{\prime}\right)$-threshold structure $\Gamma^{\prime} \in \mathcal{U}_{1}^{-}$defined on $\mathcal{P}^{\prime} \subseteq \mathcal{P}$, $H\left(b_{\Gamma^{\prime}}\right) \geq n^{\prime} H\left(s^{\prime}\right)$.

Proof: Let $\Gamma^{\prime} \in \mathcal{U}_{1}^{-}$be a $\left(1, n^{\prime}\right)$-threshold structure on $\mathcal{P}^{\prime}$. Since $\Gamma^{\prime} \in \mathcal{U}_{1}^{-}$we have $n-n^{\prime}>k-1$. Let $X \subseteq \mathcal{P} \backslash \mathcal{P}^{\prime}$ be a set of size $k-1$, let $X^{\prime} \subseteq \mathcal{P} \backslash \mathcal{P}^{\prime}$ be a set of size 1 disjoint from $X$ and let $p \in \mathcal{P}^{\prime}$.

As in the proof of Theorem 13, $p, X$, and $X^{\prime}$ satisfy the conditions of Lemma 9. As $H(s)=H\left(s^{\prime}\right)$ and $H(p)=2 H(s)$, equality holds throughout the proof of Lemma 9. In particular

$$
\begin{aligned}
H(p \mid s X) & =H\left(s^{\prime}\right) \\
H\left(p \mid s X X^{\prime} b_{\Gamma^{\prime}}\right) & =H\left(s^{\prime}\right) \\
H\left(s^{\prime} \mid s X X^{\prime} b_{\Gamma^{\prime}}\right) & =H\left(s^{\prime}\right) .
\end{aligned}
$$

It follows from (13) that $s$ and $s^{\prime}$ are independent.
For each $\omega \in[s X]$, define a new probability distribution $\tau$ on $\left\langle s^{\prime} \mathcal{P}^{\prime} \mathcal{B}\right\rangle_{\rho}$ by $\tau=\rho_{s^{\prime}} \mathcal{P}^{\prime} \mathcal{B} \mid s X=\omega$. From (13) it follows that $H\left(s^{\prime} \mid s X\right)=H\left(s^{\prime}\right)$ and so

$$
H_{\tau}\left(s^{\prime}\right)=H\left(s^{\prime}\right)
$$

Also by (13), $H\left(s^{\prime} \mid s X b_{\Gamma^{\prime}}\right)=H\left(s^{\prime} \mid s X\right)$ and so $H_{\tau}\left(s^{\prime} \mid b_{\Gamma^{\prime}}\right)=$ $H_{\tau}\left(s^{\prime}\right)$ (see Section II-A). Since $p \in \Gamma^{\prime}$ we have $H\left(s^{\prime} \mid p s X b_{\Gamma^{\prime}}\right)=0$ and thus $H_{\tau}\left(s^{\prime} \mid p b_{\Gamma^{\prime}}\right)=0$. By Lemma 10, it follows that $H_{\tau}\left(p \mid b_{\Gamma^{\prime}}\right) \geq H_{\tau}\left(s^{\prime}\right)$ and hence, $H_{\tau}(p) \geq H_{\tau}\left(s^{\prime}\right)$. By (11) and (14) it follows that

$$
H_{\tau}(p)=H\left(s^{\prime}\right) \text {. }
$$

Let $t \in \mathcal{P}$. Now consider updating to $\Delta$, a $(1,1)$-threshold structure on $\mathcal{P}^{\prime}=\{t\}$. So $\mathcal{P} \backslash t \notin \Delta$, that is,

$$
H\left(s^{\prime} \mid(\mathcal{P} \backslash t) b_{\Delta}\right)=H\left(s^{\prime}\right) \text { and } H\left(s^{\prime} \mid \mathcal{P} b_{\Delta}\right)=0 .
$$

So by Lemma $10, H\left(t \mid(\mathcal{P} \backslash t) b_{\Delta}\right) \geq H\left(s^{\prime}\right)$. It follows that

$$
H(t \mid \mathcal{P} \backslash t) \geq H\left(s^{\prime}\right) \text { and } H\left(s^{\prime} \mid \mathcal{P} \backslash t\right)=H\left(s^{\prime}\right) .
$$

Returning to $\Gamma^{\prime}$, let $\mathcal{P}^{\prime}=\left\{p_{1}, \ldots, p_{n^{\prime}}\right\}$. Then

$$
H_{\tau}\left(p_{i} \mid p_{1}, \ldots, p_{i-1}\right) \leq H_{\tau}\left(p_{i}\right)=H\left(s^{\prime}\right)
$$

by (15). Further, for $i \geq 2$, as $p_{1} X \in \Gamma$, we have

$$
\begin{aligned}
H\left(p_{i} \mid p_{1}, \ldots, p_{i-1} s X\right) & =H\left(p_{i} \mid p_{1}, \ldots, p_{i-1} X\right) \\
& \geq H\left(s^{\prime}\right) \quad \text { by }(16) .
\end{aligned}
$$

Hence $H_{\tau}\left(p_{i} \mid p_{1}, \ldots, p_{i-1}\right)=H\left(s^{\prime}\right)$ for $i \geq 2$. For $i=1$, $H_{\tau}\left(p_{1}\right)=H\left(s^{\prime}\right)$ by (15). So

$$
H_{\tau}\left(\mathcal{P}^{\prime}\right)=\sum_{i=1}^{n^{\prime}} H_{\tau}\left(p_{i} \mid p_{1}, \ldots, p_{i-1}\right)=n^{\prime} H\left(s^{\prime}\right) .
$$

As $H\left(s \mid p_{1}, \ldots, p_{n^{\prime}} X\right)=0\left(\right.$ since $\left|p_{1} \ldots p_{n^{\prime}} X\right| \geq k$ ) we have

$$
\begin{aligned}
H\left(s^{\prime} \mid p_{1}, \ldots, p_{n^{\prime}} s X\right) & =H\left(s^{\prime} \mid p_{1}, \ldots, p_{n^{\prime}} X\right) \\
& =H\left(s^{\prime}\right) \quad \text { by }(16)
\end{aligned}
$$

and so

$$
H_{\tau}\left(s^{\prime} \mid p_{1}, \ldots, p_{n^{\prime}} X\right)=H_{\tau}\left(s^{\prime}\right) \text { by }(14) .
$$

By (12), $H\left(p \mid s X b_{\Gamma^{\prime}}\right)=H\left(s^{\prime}\right)$ and by (13), $H\left(s^{\prime} \mid s X b_{\Gamma^{\prime}}\right)=$ $H\left(s^{\prime}\right)$. Now

$$
\begin{aligned}
H\left(p \mid s^{\prime} s X b_{\Gamma^{\prime}}\right) & =H\left(s^{\prime} \mid p s X b_{\Gamma^{\prime}}\right)+H\left(p \mid s X b_{\Gamma^{\prime}}\right)-H\left(s^{\prime} \mid s X b_{\Gamma^{\prime}}\right) \\
& =0+H\left(s^{\prime}\right)-H\left(s^{\prime}\right)=0 .
\end{aligned}
$$

Thus,

$$
H_{\tau}\left(p \mid b_{\Gamma^{\prime}} s^{\prime}\right)=0
$$

Thus,

$$
\begin{aligned}
H_{\tau}\left(b_{\Gamma^{\prime}}\right) \geq & \mathrm{I}_{\tau}\left(b_{\Gamma^{\prime}} ; p_{1} \ldots p_{n^{\prime}} \mid s^{\prime}\right) \\
= & H_{\tau}\left(p_{1} \ldots p_{n^{\prime}} \mid s^{\prime}\right)-H_{\tau}\left(p_{1} \ldots p_{n^{\prime}} \mid b_{\Gamma^{\prime}} s^{\prime}\right) \\
= & H_{\tau}\left(s^{\prime} \mid p_{1} \ldots p_{n^{\prime}}\right)+H_{\tau}\left(p_{1} \ldots p_{n^{\prime}}\right) \\
& -H_{\tau}\left(s^{\prime}\right)-H_{\tau}\left(p_{1} \ldots p_{n^{\prime}} \mid b_{\Gamma^{\prime}} s^{\prime}\right) \\
= & H_{\tau}\left(p_{1} \ldots p_{n^{\prime}}\right) \\
& -\sum_{i=1}^{n^{\prime}} H_{\tau}\left(p_{i} \mid p_{1} \ldots p_{i-1} b_{\Gamma^{\prime}} s^{\prime}\right) \quad \text { by }(18) \\
= & n^{\prime} H\left(s^{\prime}\right) \quad \text { by }(17) \text { and }(19) .
\end{aligned}
$$


Thus, we have

$$
H\left(b_{\Gamma^{\prime}}\right)=\sum_{\omega \in[s X]_{\rho}} \rho_{s X}(\omega) H_{\tau}\left(b_{\Gamma^{\prime}}\right) \geq n^{\prime} H\left(s^{\prime}\right)
$$

as required.

Theorem 23: Let $\mathcal{M}=\left(s s^{\prime} \mathcal{P} \mathcal{B}, \rho\right)$ be a share minimal $(k, n)$ threshold scheme with $k<n$ that can be updated to $\mathcal{U}^{-}$. Then for any $\Gamma^{\prime} \in \mathcal{U}_{1}^{-}$, where $\Gamma^{\prime}$ is a $\left(k^{\prime}, n^{\prime}\right)$-threshold structure, $H\left(b_{\Gamma^{\prime}}\right) \geq\left(n^{\prime}-k^{\prime}+1\right) H\left(s^{\prime}\right)$.

Proof: The proof is similar to the proof of Theorem 12. If $k^{\prime}=1$, then the result is proved by Lemma 22 . Suppose $k^{\prime}>1$ and $\Gamma^{\prime}$ is defined on $\mathcal{P}^{\prime}$. Let $K \subseteq \mathcal{P}^{\prime}$ be a $\left(k^{\prime}-1\right)$-set and $\mathcal{Q}^{\prime}=\mathcal{P}^{\prime} \backslash K$. Let $\kappa \in[K]_{\rho}$ and let the probability distribution $\mu$ on $\left\langle s s^{\prime} \mathcal{Q}^{\prime} \mathcal{B}\right\rangle_{\rho}$ be defined by $\mu=\rho_{s s^{\prime} \mathcal{Q}^{\prime} \mathcal{B} \mid K=\kappa}$.

Let $\mathcal{N}=\left(s s^{\prime} \mathcal{Q}^{\prime} \mathcal{B}, \mu\right)$ be the scheme corresponding to $\mu$. In a similar way as in the proof of Theorem 12 it can be shown that $\mathcal{N}$ is a $\left(k-\left(k^{\prime}-1\right), n-\left(k^{\prime}-1\right)\right)$-threshold scheme which can be updated to $\Sigma^{\prime} \in \mathcal{U}_{1}^{-}\left(1, n-\left(k^{\prime}-1\right)\right)$, with $H_{\mu}(s)=H_{\mu}\left(s^{\prime}\right)=H\left(s^{\prime}\right)$. It can be further shown that the broadcast for $\Sigma^{\prime}$ a $(1, m)$-threshold structure on $\mathcal{R}^{\prime}$ is $b_{\Sigma}$, where $\Sigma$ is the $\left(k^{\prime}, m+\left(k^{\prime}-1\right)\right)$-threshold structure on $\mathcal{R}=\mathcal{R}^{\prime} K$. The broadcast corresponding to the $\left(1, n^{\prime}-\left(k^{\prime}-1\right)\right)$-threshold structure on $\mathcal{Q}^{\prime}$ is thus $b_{\Gamma^{\prime}}$. By Lemma 22 we have

$$
H_{\mu}\left(b_{\Gamma^{\prime}}\right) \geq\left(n^{\prime}-\left(k^{\prime}-1\right)\right) H_{\mu}(s) .
$$

However, by definition

$$
H\left(b_{\Gamma^{\prime}}\right)=\sum_{\kappa \in[K]} \rho_{K}(\kappa) H_{\mu}\left(b_{\Gamma^{\prime}}\right) .
$$

Thus, $H\left(b_{\Gamma^{\prime}}\right) \geq\left(n^{\prime}-k^{\prime}+1\right) H(s)$, as required.

Decreasing the Threshold: Updating to $\mathcal{U}_{2}^{-}$: We now prove the complementary result to Theorem 23 . Thus, we consider schemes for updating to $\mathcal{U}^{-}$but will only be concerned with how big the broadcast size is in the case that the new threshold parameters belong to $\mathcal{U}_{2}^{-}$.

Theorem 24: Let $\mathcal{M}=\left(s s^{\prime} \mathcal{P} \mathcal{B}, \rho\right)$ be a share minimal $(k, n)$ threshold scheme with $k<n$ that can be updated to $\mathcal{U}^{-}$. Then, for any $\Gamma^{\prime} \in \mathcal{U}_{2}^{-}$, where $\Gamma^{\prime}$ is a $\left(k^{\prime}, n^{\prime}\right)$-threshold structure, we have

$$
H\left(b_{\Gamma^{\prime}}\right) \geq\left(\min \left(k, n^{\prime}\right)-k^{\prime}+1\right) H(s) .
$$

Proof: We divide the proof into three steps. In Step 1, we prove that for $A \subseteq \mathcal{P}$

$$
H(A)=(\min (k,|A|)+|A|) H(s) .
$$

In Step 2, for $\mathcal{M}$ as in the theorem and $\Gamma^{\prime} \in \mathcal{U}_{2}^{-}(k, n)$ with $\Gamma^{\prime}$ a $\left(1, n^{\prime}\right)$-threshold structure (so $k-1 \geq n-n^{\prime}$ ), we will prove that

$$
H\left(b_{\Gamma^{\prime}}\right) \geq \min \left(k, n^{\prime}\right) H(s) .
$$

In Step 3, we complete the proof of the theorem.

We begin our proof. We note that since $\mathcal{M}$ can be updated to $\mathcal{U}^{-},(16)$ from the proof of Lemma 22 is valid. That is,

$$
H(t \mid \mathcal{P} \backslash t) \geq H\left(s^{\prime}\right) \text { and } H\left(s^{\prime} \mid \mathcal{P} \backslash t\right)=H\left(s^{\prime}\right) .
$$

Step 1. We first show that for any $a$-set $A$ of $\mathcal{P}$, with $a \leq k$

$$
H(A)=2 a H(s) .
$$

We will do this by induction on $k$ on the class of share minimal $(k, n)$-threshold schemes with $k<n$ which can be updated to $\mathcal{U}^{-}(k, n)$. If $k=1$ then $H(p)=2 H(s)$ for $p \in \mathcal{P}$ as the scheme is share-minimal. Our inductive hypothesis will be that (23) holds for all $(\ell, n)$-threshold schemes with $1 \leq \ell \leq k$.

For a $(k, n)$-threshold scheme $\left(s s^{\prime} \mathcal{P B}, \rho\right)$ with $1<k<$ $n$, let $p \in \mathcal{P}$ and let $\pi \in[p]_{\rho}$. Define $\omega$ on $\left\langle s s^{\prime}(\mathcal{P} \backslash p) \mathcal{B}\right\rangle$ by $\omega=\rho_{s s^{\prime}(\mathcal{P} \backslash p) \mathcal{B} \mid p=\pi}$.

It can be shown that $\left(s s^{\prime}(\mathcal{P} \backslash p) \mathcal{B}, \omega\right)$ is a share-minimal $(k-1, n-1)$-threshold scheme with $1 \leq k-1 \leq n-1$, which can be updated to $\mathcal{U}^{-}(k-1, n-1)$. By inductive hypothesis, $H_{\omega}\left(A^{\prime}\right)=2(a-1) H_{\omega}(s)$ for any set $A^{\prime}$ of $\mathcal{P} \backslash p$ of size $a-1$ $(1 \leq a \leq k)$. But $H_{\omega}\left(A^{\prime}\right)=H\left(A^{\prime} \mid p=\pi\right)$, so

$$
H\left(A^{\prime} \mid p\right)=H_{\omega}\left(A^{\prime}\right)=2(a-1) H(s)
$$

Hence,

$H\left(A^{\prime} p\right)=H\left(A^{\prime} \mid p\right)+H(p)=2(a-1) H(s)+2 H(s)=2 a H(s)$

proving (23).

Now let $X$ be a $(k-1)$-set, and let $p, q \in \mathcal{P} \backslash X$ with $p \neq q$. Thus, $H(s \mid p X)=H(s \mid q X)=0$ and $H(s \mid X)=H(s)$. However, we have

$$
\begin{aligned}
0 \leq I(p ; q \mid s X) & =H(p \mid s X)-H(p \mid q s X) \\
& =H(p s X)-H(s X)-H(p \mid q X)
\end{aligned}
$$

(as $H(s \mid q X)=0$ ), which is equal to

$$
\begin{aligned}
H(s \mid p X) & +H(p X)-H(s \mid X)-H(X)-H(p \mid q X) \\
& =0+2 k H(s)-H(s)-2(k-1) H(s)-H(p \mid q X)
\end{aligned}
$$

by (23). Hence,

$$
H(p \mid q X) \leq H(s) .
$$

We now prove (20). Let $A \subseteq \mathcal{P}$. If $|A| \leq k$ then (20) holds by (23). So suppose $|A|=a>k$. Write $A=K \cup(A \backslash K)$ for a $k$-subset $K$ of $A$. Let $A \backslash K=\left\{p_{1}, \ldots, p_{a-k}\right\}$. By (22) and (24)

$$
H(s) \leq H\left(p_{i} \mid p_{1} \cdots p_{i-1} K\right) \leq H(s)
$$

for $1 \leq i \leq a-k$, and so

$$
\begin{aligned}
H(A) & =H(K)+\sum_{i=1}^{a-k} H\left(p_{i} \mid p_{1} \cdots p_{i-1} K\right) \\
& =(2 k+(a-k)) H(s) \\
& =(k+a) H(s)
\end{aligned}
$$

proving (20) for the case $|A|=a>k$.

Step 2. Let $\mathcal{M}$ be as in the theorem and let $\Gamma^{\prime} \in \mathcal{U}_{2}^{-}(k, n)$ be a $\left(1, n^{\prime}\right)$-threshold structure on $\mathcal{P}^{\prime} \subseteq \mathcal{P}$, so $k-1 \geq n-n^{\prime}$. Further

$$
\begin{aligned}
H\left(s^{\prime} \mid p b_{\Gamma^{\prime}}\right) & =0, \quad \text { for all } p \in \mathcal{P}^{\prime} \\
H\left(s^{\prime} \mid b_{\Gamma^{\prime}}\right) & =H\left(s^{\prime}\right) .
\end{aligned}
$$


Now

$$
\begin{aligned}
H\left(p \mid b_{\Gamma^{\prime}} s^{\prime}\right) & =H\left(s^{\prime} \mid p b_{\Gamma^{\prime}}\right)+H\left(p b_{\Gamma^{\prime}}\right)-H\left(b_{\Gamma^{\prime}} s^{\prime}\right) \\
& \leq 0+\left(H(p)+H\left(b_{\Gamma^{\prime}}\right)\right)-\left(H\left(b_{\Gamma^{\prime}}\right)+H(s)\right)
\end{aligned}
$$

by (25) and (26). Hence,

$$
H\left(p \mid b_{\Gamma^{\prime}} s^{\prime}\right)=H(p)-H\left(s^{\prime}\right)=H\left(s^{\prime}\right) .
$$

If $n^{\prime}=n$ let $Q=\mathcal{P}^{\prime} \backslash p$ for some $p \in \mathcal{P}^{\prime}$, otherwise, $n^{\prime}<n$ and let $Q=\mathcal{P}^{\prime}$. Now

$$
\begin{aligned}
H\left(b_{\Gamma^{\prime}}\right) \geq H\left(b_{\Gamma^{\prime}} \mid s^{\prime}\right) & =H\left(b_{\Gamma^{\prime}} \mid s^{\prime} Q\right)+I\left(b_{\Gamma^{\prime}} ; Q \mid s^{\prime}\right) \\
& \geq I\left(b_{\Gamma^{\prime}} ; Q \mid s^{\prime}\right) \\
& =H\left(Q \mid s^{\prime}\right)-H\left(Q \mid b_{\Gamma^{\prime}} s^{\prime}\right) .
\end{aligned}
$$

Now $H\left(Q \mid s^{\prime}\right)=H(Q)$ by (22), and by (20) we have $H(Q)=$ $(\min (k,|Q|)+|Q|) H(s)$. Further, if $Q=\left\{p_{1}, \ldots, p_{a}\right\}$, then

$$
\begin{aligned}
H\left(Q \mid b_{\Gamma^{\prime}} s^{\prime}\right) & =\sum_{i=1}^{a} H\left(p_{i} \mid p_{1} \cdots p_{i-1} b_{\Gamma^{\prime}} s^{\prime}\right) \\
& \leq \sum_{i=1}^{a} H\left(p_{i} \mid b_{\Gamma^{\prime}} s^{\prime}\right) \\
& \leq a H\left(s^{\prime}\right) \quad \text { by }(27) .
\end{aligned}
$$

Combining (28) and (29)

$$
H\left(b_{\Gamma^{\prime}}\right) \geq(\min (k, a)+a-a) H\left(s^{\prime}\right)=\min (k, a) H\left(s^{\prime}\right) .
$$

Now if $n^{\prime}<n$ then $a=n^{\prime}$. Otherwise, $n^{\prime}=n$ and, as $k \leq$ $n, \min (k, a)=k$. Thus, $H\left(b_{\Gamma^{\prime}}\right) \geq \min \left(k, n^{\prime}\right) H\left(s^{\prime}\right)$, proving (21).

Step 3. We now let $\Gamma^{\prime} \in \mathcal{U}_{2}^{-}(k, n)$ be a $\left(k^{\prime}, n^{\prime}\right)$-threshold structure. If $k^{\prime}=1$ the result follows by (21). Suppose $k^{\prime}>1$. We proceed in a similar manner as in the proof of Theorem 23. Let $K \subseteq \mathcal{P}^{\prime}$ be a $\left(k^{\prime}-1\right)$-set and let $\kappa \in[K]_{\rho}$. Let probability distribution $\mu$ on $\left\langle s s^{\prime}(\mathcal{P} \backslash K) \mathcal{B}\right\rangle_{\rho}$ be defined by $\mu=$ $\rho_{s s^{\prime}(\mathcal{P} \backslash K) \mathcal{B} \mid K=\kappa}$. It can be shown that $\mathcal{N}=\left(s s^{\prime}(\mathcal{P} \backslash K) \mathcal{B}, \mu\right)$ is a share minimal $\left(k-\left(k^{\prime}-1\right), n-\left(k^{\prime}-1\right)\right)$-threshold scheme which can be updated to $\mathcal{U}_{2}^{-}\left(k-\left(k^{\prime}-1\right), n-\left(k^{\prime}-1\right)\right)$ with $H_{\mu}(s)=H_{\mu}\left(s^{\prime}\right)=H(s)$. For

$$
\Sigma^{\prime} \in \mathcal{U}_{2}^{-}\left(k-\left(k^{\prime}-1\right), n-\left(k^{\prime}-1\right)\right)
$$

where $\Sigma^{\prime}$ is $(l, m)$-threshold on $\mathcal{Q}^{\prime}$, the broadcast is $b_{\Sigma^{\prime}}$ where $\Sigma^{\prime}$ is the $\left(l+\left(k^{\prime}-1\right), m+\left(k^{\prime}-1\right)\right)$ access structure on $\mathcal{Q}^{\prime} K$. Hence, $b_{\Gamma^{\prime}}$ is the broadcast in $\mathcal{N}$ for $\Pi$, where $\Pi$ is the $\left(1, n^{\prime}-\right.$ $\left.\left(k^{\prime}-1\right)\right)$-threshold structure on $\mathcal{P}^{\prime} \backslash K$. By (21) we have

$$
\begin{aligned}
H_{\mu}\left(b_{\Gamma^{\prime}}\right) & \geq \min \left(k-\left(k^{\prime}-1\right), n^{\prime}-\left(k^{\prime}-1\right)\right) H_{\mu}\left(s^{\prime}\right) \\
& \geq\left(\min \left(k, n^{\prime}\right)-k^{\prime}+1\right) H_{\mu}\left(s^{\prime}\right) .
\end{aligned}
$$

Now

$$
H\left(b_{\Gamma^{\prime}}\right)=\sum_{\kappa \in[K]} \rho_{K}(\kappa) H_{\mu}\left(b_{\Gamma^{\prime}}\right)
$$

so $H\left(b_{\Gamma^{\prime}}\right) \geq\left(\min \left(k, n^{\prime}\right)-k^{\prime}+1\right) H\left(s^{\prime}\right)$, proving the theorem.

The bound in Theorem 14 now follows immediately from Theorems 23 and 24.

\section{ACKNOWLEDGMENT}

We would like to thank the anonymous referees for their helpful comments and suggestions.

\section{REFERENCES}

[1] S. G. Barwick, W.-A Jackson, K. M. Martin, and C. M. O'Keefe Optimal Updating of Ideal Threshold Schemes. [Online]. Available: http://www.maths.adelaide.edu.au/groups/iga/preprints.html

[2] S. G. Barwick, W.-A. Jackson, K. M. Martin, and P. R. Wild, "Size of broadcast in threshold schemes with disenrollment," in Information Security and Privacy, (Lecture Notes in Computer Science). Berlin, Germany: Springer-Verlag, 2002, vol. 2384, pp. 71-88.

[3] G. R. Blakley, "Safeguarding cryptographic keys," in Proc. AFIPS 1979 Nat. Computer Conf., vol. 48, 1979, pp. 313-317.

[4] B. Blakley, G. R. Blakley, A. Chan, and J. Massey, "Threshold schemes with disenrollment," in Advances in Cryptology-CRYPTO'92 (Lecture Notes in Computer Science). Berlin, Germany: Springer-Verlag, 1993 , vol. 740, pp. 540-548.

[5] C. Blundo, A. Cresti, A. De Santis, and U. Vaccaro, "Fully dynamic secret sharing schemes," in Advances in Cryptology-CRYPTO'93 (Lecture Notes in Computer Science). Berlin, Germany: Springer-Verlag, 1994, vol. 773, pp. 110-125.

[6] C. Blundo, A. De Santis, G. Di Crescenzo, A. G. Gaggia, and U. Vaccaro, "Multi-secret sharing schemes," in Advances in Cryptology-CRYPTO'94 (Lecture Notes in Computer Science). Berlin, Germany: Springer-Verlag, 1994, vol. 839, pp. 150-163.

[7] L. Chen, D. Gollmann, and C. J. Mitchell, "Key escrow in mutually mistrusting domains," in Security Protocols (Lecture Notes in Computer Science). Berlin, Germany: Springer-Verlag, 1997, vol. 1189, pp. 139-153.

[8] T. M. Cover and J. A. Thomas, Elements of Information Theory. New York: Wiley, 1991.

[9] Y. Desmedt and S. Jajodia, "Redistributing secret shares to new access structures and its applications," George Mason Univ., Fairfax, VA, Tech. Rep. ISSE-TR-97-01, 1997.

[10] A. Herzberg, S. Jarecki, H. Krawczyk, and M. Yung, "Proactive secret sharing or: How to cope with perpetual leakage," in Advances in Cryptology-CRYPTO'95 (Lecture Notes in Computer Science). Berlin, Germany: Springer-Verlag, 1995, vol. 963, pp. 339-352.

[11] J. W. P. Hirschfeld, Projective Geometries over Finite Fields. Oxford, U.K.: Clarendon, 1979.

[12] W.-A. Jackson and K. M. Martin, "Perfect secret sharing schemes on five participants," Des., Codes, Cryptogr., vol. 9, pp. 267-286, 1996.

[13] W.-A. Jackson, K. M. Martin, and C. M. O'Keefe, "Geometrical contributions to secret sharing theory," J. Geom., vol. 79, no. 1-2, pp. 102-133, 2004.

[14] E. Karnin, J. Greene, and M. Hellman, "On secret sharing systems," IEEE Trans. Inf. Theory, vol. IT-29, no. 1, pp. 35-41, Jan. 1983.

[15] K. M. Martin, "Untrustworthy participants in secret sharing schemes," in Cryptography and Coding III. Oxford, U.K.: Oxford Univ. Press, 1993, pp. 255-264.

[16] K. M. Martin, R. Safavi-Naini, and H. Wang, "Bounds and techniques for efficient redistribution of secret shares to new access structures," Comput. J., vol. 42, no. 8, pp. 638-649, 1999.

[17] A. Shamir, "How to share a secret," Commun. ACM, vol. 22, no. 11, pp. 612-613, 1979.

[18] G. J. Simmons, "Prepositioned shared secret and/or shared control schemes," in Advances in Cryptology-EUROCRYPT'89 (Lecture Notes in Computer Science). Berlin, Germany: Springer-Verlag, 1990, vol. 434, pp. 436-467.

[19] _ " "An introduction to shared secret and/or shared control schemes and their applications," in Contemporary Cryptology. New York: IEEE Press, 1992, pp. 441-497.

[20] D. R. Stinson, "An explication of secret sharing schemes," Des., Codes, Cryptogr., vol. 2, pp. 357-390, 1992. 